TRANSACTIONS OF THE

AMERICAN MATHEMATICAL SOCIETY

Volume 354, Number 8, Pages 3155-3179

S 0002-9947(02)02982-3

Article electronically published on April 2, 2002

\title{
FORMATION AND PROPAGATION OF SINGULARITIES FOR $2 \times 2$ QUASILINEAR HYPERBOLIC SYSTEMS
}

\author{
DE-XING KONG
}

\begin{abstract}
Employing the method of characteristic coordinates and the singularity theory of smooth mappings, in this paper we analyze the long-term behaviour of smooth solutions of general $2 \times 2$ quasilinear hyperbolic systems, provide a complete description of the solution close to blow-up points, and investigate the formation and propagation of singularities for $2 \times 2$ systems of hyperbolic conservation laws.
\end{abstract}

\section{INTRODUCTION}

It is well known that smooth solutions to nonlinear hyperbolic systems generally exist in finite time even if the initial data is sufficiently smooth and small (see [1], [3] 6], 8], 10]-[11, [14, 17]). After this time, only weak solutions (usually containing shocks or other kinds of discontinuities) can be defined. The following questions arise naturally:

(I) When and where do the solutions blow up?

(II) What quantities blow up? How do they blow up?

(III) What kinds of singularities appear? How do the discontinuities, in particular, shocks grow out of nothing?

These problems are very important and interesting in the sense of both mathematics and physics. For problems (I) and (II), some methods have been established and many results have been obtained (see [1], [3], 6], [8], [10]-[11], [14], [17]). As for problem (III), since this kind of nonlinear phenomenon is too complex, up to now, only a few results on shock formation have been known. For a single conservation law, these problems have been solved well by the characteristic method (see [5], [7], [18-19]). For the $p$-system, Lebaud [13] discussed the problem of shock formation under the hypothesis that one of the Riemann invariants is a constant. Recently, in a manner similar to Lebaud's, Chen and Dong [4] generalized the result given in [13] to the case of general initial data. Bryant, Griffiths and Hsu [2] presented a geometric theory of differential equations; in particular, for a $2 \times 2$ hyperbolic

Received by the editors May 24, 2000 and, in revised form, May 4, 2001.

2000 Mathematics Subject Classification. Primary 35L45, 35L67; Secondary 35L65, 76L05.

Key words and phrases. Quasilinear hyperbolic system, smooth solution, blow-up of cusp type, shock, weak discontinuity.

The author was supported in part by the National Science Foundation of China under Grant \# 10001024 and the Special Funds for Major State Basic Research Projects of China.

(C)2002 American Mathematical Society 
system of conservation laws, they showed that the geometric solution capture 1 the shock solution if, and only if, the system is special 2 .

We consider the Cauchy problem for the general $2 \times 2$ quasilinear hyperbolic system

$$
\begin{gathered}
\left\{\begin{array}{l}
\frac{\partial w}{\partial t}+\lambda_{+}(w, z) \frac{\partial w}{\partial x}=0, \\
\frac{\partial z}{\partial t}+\lambda_{-}(w, z) \frac{\partial z}{\partial x}=0,
\end{array}\right. \\
t=0: \quad w=w_{0}(x), z=z_{0}(x),
\end{gathered}
$$

where $w$ and $z$, the so-called Riemann invariants, are the unknown functions, and $\lambda_{ \pm}(w, z)$ are $C^{1}$ functions, $w_{0}(x), z_{0}(x)$ are $C^{1}$ functions with bounded $C^{1}$ norm.

Suppose that system (1.1) is strictly hyperbolic. Since we are only interested in the solution near the blow-up point, without loss of generality, we assume that

$$
\lambda_{-}(w, z)<0<\lambda_{+}(w, z) .
$$

Suppose furthermore that each characteristic is genuinely nonlinear in the sense of Lax. Without loss of generality, we may assume that

$$
\frac{\partial \lambda_{+}(w, z)}{\partial w}>0, \quad \frac{\partial \lambda_{-}(w, z)}{\partial z}>0
$$

Remark 1.1. It is well known that, for any given quasilinear hyperbolic system

$$
\frac{\partial u_{i}}{\partial t}+\sum_{j=1}^{2} a_{i j}(u) \frac{\partial u_{j}}{\partial x}=0 \quad(i=1,2),
$$

by a suitable invertible transformation of $\left(u_{1}, u_{2}\right)$ we can always rewrite (1.5) as a system of the form (1.1), at least in a local domain, where $u=\left(u_{1}, u_{2}\right)^{\mathrm{T}}$ is the unknown function and the $a_{i j}(u)(i, j=1,2)$ are smooth given functions of $u$.

Remark 1.2. Some physical systems (for example, the system of isentropic gas dynamics) always satisfy the assumption that system (1.1) is genuinely nonlinear in the sense of Lax. If only one of the characteristics is genuinely nonlinear, then we may develop a similar theory. However, if system (1.1) is linearly degenerate in the sense of Lax, that is, $\frac{\partial \lambda_{+}(w, z)}{\partial w} \equiv 0$ and $\frac{\partial \lambda_{-}(w, z)}{\partial z} \equiv 0$, then the $C^{1}$ solution of the Cauchy problem (1.1)-(1.2) always exists globally in time (see [14]).

In this paper, we present a systematic analysis of the long-term behaviour of a smooth solution of the Cauchy problem (1.1)-(1.2); in particular, we provide a complete description of the solution close to a blow-up point. Based on this, in a way completely different from that of Lebaud [13], we give a detailed discussion of the process of shock formation in the case when system (1.1) is of a form of conservation laws. In particular, we do not need the assumption in [13] that one of the Riemann invariants is a constant.

The paper is organized as follows. In $\S 2$ we give some preliminaries. Using the method of characteristic coordinates and the singularity theory of the smooth

\footnotetext{
${ }^{1}$ For a scalar conservation law, the shock solution arises by taking a suitable cross-section of the geometric solution. In this sense, we say the geometric solution captures the shock solution.

${ }^{2}$ Unfortunately, according to the definition of special system in [2], this kind of system is so special that many physical systems (for example, the system of isentropic gas dynamics) do not belong to this class.
} 
mappings, in $\S 3$ we determine exactly the blow-up time of a smooth solution of the Cauchy problem (1.1)-(1.2) and the set of all blow-up points at this time, and describe the breakdown mechanism of the smooth solution. In $\S 4$, under appropriate assumptions we first prove that the solution is a blow-up solution of cusp type, according to the terminology of Alinhac [1]; then we construct the envelope of characteristics of the same family, and show that the formation of a singularity is due to this envelope of characteristics and the singularity occurs at the starting point of the envelope, i.e., the point with minimum $t$-value on the envelope. By the way, a triple-valued solution and an artificial solution are introduced and some estimates on the blow-up rates of the solution are also given in $\S 4$. In $\S 5$ we study the $2 \times 2$ system of conservation laws. Based on $\S 4$, we construct a shock and a weak discontinuity issuing from the blow-up point, and prove the existence of a classical discontinuous solution in a local domain.

\section{Preliminaries}

In this section, we briefly recall some basic facts on the characteristic coordinates for $2 \times 2$ quasilinear hyperbolic systems.

By the existence and uniqueness of a local $C^{1}$ solution of the Cauchy problem for a quasilinear hyperbolic system (see [15]), there exists a positive number $h$ such that the Cauchy problem (1.1)-(1.2) has a unique $C^{1}$ solution $(w(t, x), z(t, x))$ on the strip $D(h)=\{(t, x) \mid 0 \leq t \leq h,-\infty<x<\infty\}$. On the existence domain of the $C^{1}$ solution, let $x=x_{+}(t, \alpha)$ (resp. $\left.x=x_{-}(t, \beta)\right)$ be the fast (resp. slow) characteristic passing through any fixed point $(0, \alpha)$ (resp. $(0, \beta))$. We have

$$
\begin{gathered}
w\left(t, x_{+}(t, \alpha)\right)=w_{0}(\alpha), \quad z\left(t, x_{-}(t, \beta)\right)=z_{0}(\beta), \\
\frac{d x_{+}(t, \alpha)}{d t}=\lambda_{+}\left(w_{0}(\alpha), z\left(t, x_{+}(t, \alpha)\right)\right), \quad x_{+}(0, \alpha)=\alpha, \\
\frac{d x_{-}(t, \beta)}{d t}=\lambda_{-}\left(w\left(t, x_{-}(t, \beta)\right), z_{0}(\beta)\right), \quad x_{-}(0, \beta)=\beta .
\end{gathered}
$$

It follows from (2.1) that on the existence domain of the $C^{1}$ solution we have

$$
\begin{gathered}
\underline{m}_{1} \leq w(t, x) \leq \bar{m}_{1}, \quad \underline{m}_{2} \leq z(t, x) \leq \bar{m}_{2}, \\
0<M_{\star} \leq-\lambda_{-}(w, z), \quad \lambda_{+}(w, z) \leq M^{\star}<\infty,
\end{gathered}
$$

where $\underline{m}_{1}=\inf _{x \in \boldsymbol{R}}\left\{w_{0}(x)\right\}, \bar{m}_{1}=\sup _{x \in \boldsymbol{R}}\left\{w_{0}(x)\right\}, \underline{m}_{2}=\inf _{x \in \boldsymbol{R}}\left\{z_{0}(x)\right\}, \bar{m}_{2}=$ $\sup _{x \in \boldsymbol{R}}\left\{z_{0}(x)\right\}, M_{\star}=\inf _{\left(\nu_{1}, \nu_{2}\right) \in\left[\underline{m}_{1}, \bar{m}_{1}\right] \times\left[\underline{m}_{2}, \bar{m}_{2}\right]}\left\{-\lambda_{-}\left(\nu_{1}, \nu_{2}\right), \lambda_{+}\left(\nu_{1}, \nu_{2}\right)\right\}, M^{\star}=$ $\sup _{\left(\nu_{1}, \nu_{2}\right) \in\left[\underline{m}_{1}, \bar{m}_{1}\right] \times\left[\underline{m}_{2}, \bar{m}_{2}\right]}\left\{-\lambda_{-}\left(\nu_{1}, \nu_{2}\right), \lambda_{+}\left(\nu_{1}, \nu_{2}\right)\right\}$.

Remark 2.1. (2.4) implies that the solution itself always remains bounded on the existence domain of the $C^{1}$ solution. Hence, if the $C^{1}$ solution blows up at a finite time, then the first order derivatives of the solution must tend to the infinity at the blow-up point.

Choose the characteristic coordinates $\alpha$ and $\beta$ in the following way (see 9] or $\S 4$ of Chapter 2 in [14]): on any fixed fast (resp. slow) characteristic, $\alpha$ (resp. $\beta$ ) is taken as the $x$-coordinate of the intersection point of this characteristic with the 
$x$-axis. It is easy to see that the half-plane $t \geq 0$ in the $(t, x)$-plane reduces to the half-plane $\alpha \leq \beta$ in the $(\alpha, \beta)$-plane, and

$$
w=w_{0}(\alpha), \quad z=z_{0}(\beta)
$$

It turns out that the original Cauchy problem (1.1)-(1.2) reduces to

$$
\begin{aligned}
& \left\{\begin{array}{l}
\frac{\partial x}{\partial \alpha}=\lambda_{-}\left(w_{0}(\alpha), z_{0}(\beta)\right) \frac{\partial t}{\partial \alpha}, \quad \forall \alpha<\beta, \\
\frac{\partial x}{\partial \beta}=\lambda_{+}\left(w_{0}(\alpha), z_{0}(\beta)\right) \frac{\partial t}{\partial \beta},
\end{array}\right. \\
& \alpha=\beta: t=0, x=\beta .
\end{aligned}
$$

Obviously, (2.7)-(2.8) is a Cauchy problem for a linear hyperbolic system; it always has a unique global $C^{1}$ solution $(t(\alpha, \beta), x(\alpha, \beta))$ on the half-plane $\alpha \leq \beta$. Let $\Pi$ be the mapping defined by the solution $(t(\alpha, \beta), x(\alpha, \beta))$ and $J(\alpha, \beta)$ be the Jacobian $\frac{\partial(t, x)}{\partial(\alpha, \beta)}$ of $\Pi$. Noting $(2.7)$, we have

$$
\begin{aligned}
J(\alpha, \beta) & =\left(\lambda_{+}\left(w_{0}(\alpha), z_{0}(\beta)\right)-\lambda_{-}\left(w_{0}(\alpha), z_{0}(\beta)\right)\right) t_{\alpha}(\alpha, \beta) t_{\beta}(\alpha, \beta) \\
& =\left(\left(\lambda_{-}\left(w_{0}(\alpha), z_{0}(\beta)\right)\right)^{-1}-\left(\lambda_{+}\left(w_{0}(\alpha), z_{0}(\beta)\right)\right)^{-1}\right) x_{\alpha}(\alpha, \beta) x_{\beta}(\alpha, \beta) .
\end{aligned}
$$

Remark 2.2. It is easy to see that if $\alpha$ and $\beta$ are the characteristic coordinates, then $\tilde{\alpha}=f(\alpha)$ and $\tilde{\beta}=g(\beta)$ can also be taken as the characteristic coordinates, where $f$ and $g$ are arbitrarily given $C^{1}$ functions with $f^{\prime} \neq 0$ and $g^{\prime} \neq 0$. By suitably choosing the characteristic coordinates, we can get the corresponding problem on the $(\tilde{\alpha}, \tilde{\beta})$-plane in a simpler form.

Combining Theorem 2.2 and Theorem 4.1 in Chapter 2 of [14] gives

Theorem 2.1. Under the assumptions (1.3)-(1.4), the Cauchy problem (1.1)-(1.2) admits a unique global $C^{1}$ solution on $t \geq 0$ if and only if

$$
w_{0}^{\prime}(x) \geq 0, \quad z_{0}^{\prime}(x) \geq 0, \quad \forall x \in \mathbb{R} ;
$$

or equivalently

$$
\frac{\partial t}{\partial \alpha}(\alpha, \beta)<0, \quad \frac{\partial t}{\partial \beta}(\alpha, \beta)>0, \quad \forall \alpha \leq \beta
$$

\section{General Blow-up}

In this section, we determine exactly the blow-up time of a smooth solution and the set of all blow-up points at this time, and describe the breakdown mechanism of the $C^{1}$ solution.

By Theorem 2.1, the $C^{1}$ solution of the Cauchy problem (1.1)-(1.2) blows up in a finite time if and only if the initial data is non-increasing. Therefore, throughout this paper, we assume that

(H1) there exists a $x_{*} \in \mathbb{R}$ such that $w_{0}^{\prime}\left(x_{*}\right)<0$ or $z_{0}^{\prime}\left(x_{*}\right)<0$.

Thus, noting Theorem 2.1 again, we have

$$
\begin{gathered}
J^{-1}(0) \triangleq\{(\alpha, \beta) \mid \alpha \leq \beta, J(\alpha, \beta)=0\} \neq \emptyset, \\
0<t_{0} \triangleq \inf _{(\alpha, \beta) \in J^{-1}(0)}\{t(\alpha, \beta)\}<\infty .
\end{gathered}
$$

By a standard method (see [14]), we can easily prove the following lemma. 
Lemma 3.1. Under the assumptions (1.3)-(1.4) and (H1), the Cauchy problem (1.1)-(1.2) has a unique $C^{1}$ solution $(w(t, x), z(t, x))$ in the domain $D\left(t_{0}\right) \triangleq\{(t, x) \mid$ $\left.0 \leq t<t_{0},-\infty<x<\infty\right\}$. Moreover,

$$
\limsup _{t \nearrow t_{0}}\left\{\left\|w_{x}(t, \cdot)\right\|_{C^{0}}+\left\|z_{x}(t, \cdot)\right\|_{C^{0}}\right\}=\infty .
$$

Lemma 3.1 implies that $t_{0}$ is just the blow-up time, i.e., the life span of the $C^{1}$ solution of the Cauchy problem (1.1)-(1.2). There are only two possibilities:

(i) the solution blows up only at $\left(t_{0},+\infty\right)$ or $\left(t_{0},-\infty\right)$ or $\left(t_{0}, \pm \infty\right)$;

(ii) the solution blows up at certain points $\left(t_{0}, x\right)$, where $x \in \mathbb{R}$.

Since possibility (i) is an extreme case, in this paper we do not consider it. In order to avoid this possibility, we furthermore assume that there exists at least one point $\left(\alpha_{0}, \beta_{0}\right) \in J^{-1}(0)$ such that $t\left(\alpha_{0}, \beta_{0}\right)=t_{0}$, namely,

$$
J^{-1}(0) \cap t^{-1}\left(t_{0}\right) \neq \emptyset \text {. }
$$

Remark 3.1 (Discussion of hypothesis (H2)). (H2) is a very general and weak assumption. For example, it is easy to show that if the initial data is periodic, or has compact support, or satisfies $\lim _{x \rightarrow \pm \infty}\left\{w_{0}^{\prime}(x), z_{0}^{\prime}(x)\right\} \rightarrow 0$, then the assumption (H2) is automatically valid. On the other hand, if we do not require (H2), then we can construct a counterexample such that the blow-up points of the solution are $\left(t_{0}, \pm \infty\right)$ instead of $\left(t_{0}, x\right)$, where $x$ is an arbitrary real number. So the assumption (H2) is essential. That is to say, if we do not assume (H2), then the blow-up time may still be $t_{0}$, but the solution does not blow up at any point $\left(t_{0}, x\right)$.

Let

$$
\mathcal{S}=\left\{\left(t_{0}, x\right) \mid x=x(\alpha, \beta), \quad \forall(\alpha, \beta) \in J^{-1}(0) \cap t^{-1}\left(t_{0}\right)\right\} .
$$

By (H2), there exists at least one point $\left(t_{0}, x_{0}\right)$ such that

$$
\left(t_{0}, x_{0}\right) \in \mathcal{S} \neq \emptyset
$$

where $t_{0}=t\left(\alpha_{0}, \beta_{0}\right), x_{0}=x\left(\alpha_{0}, \beta_{0}\right)$, with $\left(\alpha_{0}, \beta_{0}\right) \in J^{-1}(0) \cap t^{-1}\left(t_{0}\right)$.

In Theorem 3.2 we will show that the derivatives of a solution $(w(t, x), z(t, x))$ blow up at $\left(t_{0}, x\right)$ if and only if $\left(t_{0}, x\right) \in \mathcal{S}$. Therefore, $\mathcal{S}$ is called the set of all blow-up points on $t=t_{0}$. Obviously, such blow-up points need not be unique.

For an arbitrary point $\left(t_{0}, x_{0}\right) \in \mathcal{S}$, it follows from the definition of $\mathcal{S}$ that there exists at least one point $\left(\alpha_{0}, \beta_{0}\right)$ such that $\left(\alpha_{0}, \beta_{0}\right) \in J^{-1}(0) \cap t^{-1}\left(t_{0}\right) \cap x^{-1}\left(x_{0}\right)$. Such a point $\left(\alpha_{0}, \beta_{0}\right)$ is called the preimage of the blow-up point $\left(t_{0}, x_{0}\right)$. Clearly, the preimage of $\left(t_{0}, x_{0}\right)$ need not be unique.

Theorem 3.1. For any fixed point $\left(t_{0}, x_{0}\right) \in \mathcal{S}$, there exist two real numbers $\alpha_{\min }$ and $\alpha_{\max }, \alpha_{\min } \leq \alpha_{\max }$ (resp. $\beta_{\min }$ and $\beta_{\max }, \beta_{\min } \leq \beta_{\max }$ ) such that all fast (resp. slow) characteristics $x=x_{+}(t, \alpha)$ (resp. $x=x_{-}(t, \beta)$ ) reach $\left(t_{0}, x_{0}\right)$ when $t \nearrow t_{0}$, where $t \in\left[0, t_{0}\right)$ and $\alpha \in\left[\alpha_{\min }, \alpha_{\max }\right]$ (resp. $\beta \in\left[\beta_{\min }, \beta_{\max }\right]$ ). Moreover, any preimage $\left(\alpha_{0}, \beta_{0}\right)$ of $\left(t_{0}, x_{0}\right)$ has the following properties:

1) $\alpha_{0} \in\left[\alpha_{\min }, \alpha_{\max }\right], \quad \beta_{0} \in\left[\beta_{\min }, \beta_{\max }\right]$;

2) $J(\alpha, \beta)<0, \quad \forall(\alpha, \beta) \in\left\{(\alpha, \beta) \mid \alpha_{0} \leq \alpha \leq \beta \leq \beta_{0}\right\} \backslash\left\{\left(\alpha_{0}, \beta_{0}\right)\right\}$.

We refer to $x=x_{+}\left(t, \alpha_{\max }\right)$ (resp. $\left.x=x_{+}\left(t, \alpha_{\min }\right)\right)$ as the rightmost (resp. leftmost) fast characteristic passing through $\left(t_{0}, x_{0}\right)$, and to $x=x_{-}\left(t, \beta_{\max }\right)$ (resp. 
$\left.x=x_{-}\left(t, \beta_{\min }\right)\right)$ as the rightmost (resp. leftmost) slow characteristic passing through $\left(t_{0}, x_{0}\right)$.

Proof of Theorem 3.1. For any fixed point $\left(t, x_{0}\right)$ (where $t \in\left[0, t_{0}\right)$ ), we can draw a unique fast characteristic $x=x_{+}(s, \alpha(t))$, where $\alpha(t)$ is the $x$-coordinate of the intersection point of this characteristic with the $x$-axis. It is easy to show that $\{\alpha(t)\}$ is bounded and strictly decreasing when $t \nearrow t_{0}$. Therefore, $\lim _{t / t_{0}} \alpha(t)$ exists; we denote it by $\alpha$. Obviously, $x=x_{+}(s, \alpha)$ (where $s \in\left[0, t_{0}\right)$ ) is a fast characteristic passing through $\left(t_{0}, x_{0}\right)$. Such fast characteristics need not be unique, because we can draw them in other ways. Defining the maximum (resp. minimum) of all such $\alpha$ as $\alpha_{\max }$ (resp. $\alpha_{\min } \sqrt{3}$, we can easily prove that $\alpha_{\max }$ and $\alpha_{\min }$ are just the desired real numbers. In a similar way, we can define $\beta_{\max }$ and $\beta_{\min }$.

The rest of the proof is obvious.

Theorem 3.2. Under the assumptions (1.3)-(1.4) and (H1), the $C^{1}$ solution (w,z) of the Cauchy problem (1.1)-(1.2) must blow up in a finite time, and the life span of the solution is just $t_{0}$. Furthermore, if (H2) is assumed, then $\mathcal{S} \neq \emptyset$, and it is the set of all blow-up points on the line $t=t_{0}$; that is to say, the solution blows up at $\left(t_{0}, x\right)$ if and only if $\left(t_{0}, x\right) \in \mathcal{S}$. More precisely, for any fixed $\left(t_{0}, x_{0}\right) \in \mathcal{S}$ there exists at least one preimage $\left(\alpha_{0}, \beta_{0}\right)$ such that either $(a) x_{\alpha}\left(\alpha_{0}, \beta_{0}\right)=0$-then along a fast characteristic $x=x_{+}(t, \alpha)$, where $\alpha \in\left[\alpha_{\min }, \alpha_{\max }\right]$,

$$
w_{x}(t, x) \longrightarrow-\infty \text { as } t \nearrow t_{0}
$$

or (b) $x_{\beta}\left(\alpha_{0}, \beta_{0}\right)=0$-then along a slow characteristic $x=x_{-}(t, \beta)$, where $\beta \in$ $\left[\beta_{\min }, \beta_{\max }\right]$,

$$
z_{x}(t, x) \longrightarrow-\infty \quad \text { as } t \nearrow t_{0}
$$

For any fixed $\beta \in \mathbb{R}$, we consider the characteristic $x=x_{-}(t, \beta)$ passing through $(0, \beta)$, where $t \in\left[0, t_{0}\right)$. Passing through any point $\left(t, x_{-}(t, \beta)\right)$ on $x=x_{-}(t, \beta)$, we draw a unique fast characteristic and denote the intersection point of this characteristic with the $x$-axis by $(0, \alpha)$. Then

$$
t=t(\alpha, \beta), \quad x_{-}(t, \beta)=x(\alpha, \beta), \quad \forall(\alpha, \beta) \in \Omega\left(t_{0}\right),
$$

where $(t(\alpha, \beta), x(\alpha, \beta))$ is the $C^{1}$ solution of the Cauchy problem (2.7)-(2.8) and $\Omega\left(t_{0}\right) \triangleq\left\{(\alpha, \beta) \mid \alpha \leq \beta, t(\alpha, \beta)<t_{0}\right\}$.

In order to prove Theorem 3.2, we need the following lemma.

Lemma 3.2. We have

$$
\frac{\partial x_{-}(t, \beta)}{\partial \beta}=\left(1-\frac{\lambda_{-}\left(w_{0}(\alpha), z_{0}(\beta)\right)}{\lambda_{+}\left(w_{0}(\alpha), z_{0}(\beta)\right)}\right) x_{\beta}(\alpha, \beta), \quad \forall(\alpha, \beta) \in \Omega\left(t_{0}\right) .
$$

Similarly,

$$
\frac{\partial x_{+}(t, \alpha)}{\partial \alpha}=\left(1-\frac{\lambda_{+}\left(w_{0}(\alpha), z_{0}(\beta)\right)}{\lambda_{-}\left(w_{0}(\alpha), z_{0}(\beta)\right)}\right) x_{\alpha}(\alpha, \beta), \quad \forall(\alpha, \beta) \in \Omega\left(t_{0}\right) .
$$

Proof. Differentiating the second equality in (3.8) with respect to $\beta$ gives

$$
\frac{\partial x_{-}(t, \beta)}{\partial \beta}+\frac{d x_{-}(t, \beta)}{d t} \frac{\partial t(\alpha, \beta)}{\partial \beta}=\frac{\partial x(\alpha, \beta)}{\partial \beta} \text {. }
$$

\footnotetext{
${ }^{3}(2.5)$ guarantees that $\alpha_{\max }$ and $\alpha_{\min }$ are finite real numbers.
} 
Noting (2.3), we obtain from (3.11) that

$$
\frac{\partial x_{-}(t, \beta)}{\partial \beta}+\lambda_{-}\left(w_{0}(\alpha), z_{0}(\beta)\right) \frac{\partial t(\alpha, \beta)}{\partial \beta}=\frac{\partial x(\alpha, \beta)}{\partial \beta},
$$

where we have made use of the fact that $w$ is a constant along any fixed fast characteristic; in the present situation, $w\left(t, x_{-}(t, \beta)\right)=w_{0}(\alpha)$. Then, using the second equation in (2.7), we get the desired (3.9) immediately.

The proof of (3.10) is similar. This completes the proof.

Proof of Theorem 3.2. The first part of Theorem 3.2 is just the conclusion of Lemma 3.1. By (3.5), the fact that $\mathcal{S} \neq \emptyset$ is obvious under the assumption (H2).

We prove that for any fixed $\left(t_{0}, x_{0}\right) \in \mathcal{S}$, the derivatives of the solution must blow up at this point.

As before, let $\left(\alpha_{0}, \beta_{0}\right)$ be a preimage of $\left(t_{0}, x_{0}\right)$. Noting (1.3) and (2.9) and using the fact that $J\left(\alpha_{0}, \beta_{0}\right)=0$, we have

$$
x_{\alpha}\left(\alpha_{0}, \beta_{0}\right)=0 \quad \text { or } \quad x_{\beta}\left(\alpha_{0}, \beta_{0}\right)=0 .
$$

We now prove (3.7) under the assumption that $x_{\beta}\left(\alpha_{0}, \beta_{0}\right)=0$.

As a matter of fact, we have (see $\S 2$ in Chapter 2 of [14], or [9])

$$
\frac{\partial x_{-}(t, \beta)}{\partial \beta}=e^{A(t)}\left(1+z_{0}^{\prime}(\beta) \int_{0}^{t} \frac{\partial \lambda_{-}}{\partial z}\left(w\left(\tau, x_{-}(\tau, \beta)\right), z_{0}(\beta)\right) e^{-A(\tau)} d \tau\right),
$$

where $A(t)=H_{-}\left(w\left(t, x_{-}(t, \beta)\right), z_{0}(\beta)\right)-H_{-}\left(w_{0}(\beta), z_{0}(\beta)\right)$; here $H_{-}(w, z)$ is defined by

$$
\frac{\partial H_{-}(w, z)}{\partial w}=\frac{1}{\lambda_{-}(w, z)-\lambda_{+}(w, z)} \frac{\partial \lambda_{-}(w, z)}{\partial w} .
$$

If $x_{\beta}\left(\alpha_{0}, \beta_{0}\right)=0$, then, using (3.9), we obtain that, along $x=x_{-}\left(t, \beta_{0}\right)$,

$$
\left.\frac{\partial x_{-}(t, \beta)}{\partial \beta}\right|_{\beta=\beta_{0}} \longrightarrow 0 \quad \text { as } t \nearrow t_{0}
$$

and then, using (3.13), we observe that $z_{0}^{\prime}\left(\beta_{0}\right)<0$. Moreover, by contradiction we can prove that

$$
z_{0}^{\prime}(\beta)<0, \quad \forall \beta \in\left[\beta_{\min }, \beta_{\max }\right] .
$$

In fact, if (3.15) is not valid, then there exists at least one point $\beta_{\star} \in\left[\beta_{\min }, \beta_{\max }\right]$ such that $z_{0}^{\prime}\left(\beta_{\star}\right) \geq 0$. Thus, for any given, sufficiently small $\varepsilon>0$, there exists a $\beta_{\varepsilon} \in\left[\beta_{\min }, \beta_{\max }\right]$ such that $z_{0}^{\prime}(\beta) \geq-\varepsilon, \forall \beta \in\left[\beta_{\varepsilon}, \beta_{\star}\right]$, where, without loss of generality, we assume that $\beta_{\varepsilon}<\beta_{\star}$. Then it follows from (3.13) that

$$
\frac{\partial x_{-}(t, \beta)}{\partial \beta} \geq \mu_{1}>0, \quad \forall \beta \in\left[\beta_{\varepsilon}, \beta_{\star}\right], \quad \forall t \in\left[0, t_{0}\right),
$$

where $\mu_{1}$ is a constant independent of $t$ and $\beta$ but depending on $\varepsilon$. Hence,

$$
x_{-}\left(t, \beta_{\star}\right)-x_{-}\left(t, \beta_{\varepsilon}\right) \geq \mu_{1}\left(\beta_{\star}-\beta_{\varepsilon}\right)>0, \quad \forall t \in\left[0, t_{0}\right) .
$$

This contradicts the conclusion of Theorem 3.1.

\footnotetext{
${ }^{4}$ Here we assume that $\beta_{\min }<\beta_{\max }$, since the desired conclusion is obvious if $\beta_{\min }=\beta_{\max }$.
} 
We next prove that

$$
\frac{\partial x_{-}(t, \beta)}{\partial \beta} \longrightarrow 0 \quad \text { as } \quad t \nearrow t_{0}, \quad \forall \beta \in\left[\beta_{\min }, \beta_{\max }\right] .
$$

Let $(\alpha, \beta)$ be the characteristic coordinates corresponding to $\left(t, x_{-}(t, \beta)\right)$. Noting that $w\left(t, x_{-}(t, \beta)\right)=w_{0}(\alpha)$, we obtain from (2.3) that

$$
\frac{d x_{-}(t, \beta)}{d t}=\lambda_{-}\left(w_{0}(\alpha), z_{0}(\beta)\right), \quad \forall t \in\left[0, t_{0}\right), \quad \forall \beta \in \mathbb{R} .
$$

Differentiating (3.17) with respect to $\beta$ and noting (1.4), (3.15), we get

$$
\frac{d}{d t}\left(\frac{\partial x_{-}(t, \beta)}{\partial \beta}\right)=\frac{\partial \lambda_{-}}{\partial z} z_{0}^{\prime}(\beta)<0, \quad \forall t \in\left[0, t_{0}\right), \quad \forall \beta \in\left[\beta_{\min }, \beta_{\max }\right] .
$$

Moreover, it is easy to see that

$$
\frac{\partial x_{-}(t, \beta)}{\partial \beta}>0, \quad \forall t \in\left[0, t_{0}\right), \quad \forall \beta \in \mathbb{R} .
$$

(3.18)-(3.19) imply that $\lim _{t} / t_{0} \frac{\partial x_{-}(t, \beta)}{\partial \beta}$ exists and is non-negative for all $\beta \in$ $\left[\beta_{\min }, \beta_{\max }\right]$. As before, by contradiction we can prove that $\lim _{t} / t_{0} \frac{\partial x_{-}(t, \beta)}{\partial \beta}=0$. This proves (3.16). It is well known that (3.16) implies (3.7).

Similarly, we can prove (3.6) under the assumption that $x_{\alpha}\left(\alpha_{0}, \beta_{0}\right)=0$.

We finally show that for any fixed point $\left(t_{0}, y\right) \notin \mathcal{S}$, there exists a small half-ball $\mathcal{O}_{\left(t_{0}, y\right)}^{\varepsilon_{*}}=\left\{(t, x) \mid t<t_{0}, \sqrt{\left|t-t_{0}\right|^{2}+|x-y|^{2}}<\varepsilon_{*}\right\}$, centered at $\left(t_{0}, y\right)$, such that

$$
\left|w_{x}(t, x)\right|+\left|z_{x}(t, x)\right| \leq M, \quad \forall(t, x) \in \mathcal{O}_{\left(t_{0}, y\right)}^{\varepsilon_{*}},
$$

where $\varepsilon_{*}>0$ is a small number and $M>0$ is a constant independent of $(t, x)$.

By the definition of $\mathcal{S}$ (see $(3.4))$, if $\left(t_{0}, y\right) \notin \mathcal{S}$, then there exists a ball $B_{\left(t_{0}, y\right)}^{\tilde{\varepsilon}}=$ $\left\{(t, x) \mid \sqrt{\left|t-t_{0}\right|^{2}+|x-y|^{2}} \leq \tilde{\varepsilon}\right\}$ such that

$$
J(\alpha, \beta) \neq 0, \quad \forall(\alpha, \beta) \in \Pi^{-1}\left(B_{\left(t_{0}, y\right)}^{\tilde{\varepsilon}}\right),
$$

where $\tilde{\varepsilon}>0$ is a small number, $(\alpha, \beta)$ are the characteristic coordinates corresponding to $(t, x) \in B_{\left(t_{0}, y\right)}^{\tilde{\varepsilon}}$, and $\Pi^{-1}=\Pi^{-1}(t, x)$ stands for the inverse mapping of $\Pi=\Pi(\alpha, \beta)$ defined by the $C^{1}$ solution $(t(\alpha, \beta), x(\alpha, \beta))$ of the Cauchy problem (2.7)-(2.8). Hence, there is another small positive number $\varepsilon_{*}<\tilde{\varepsilon}$ such that

$$
x_{\alpha}(\alpha, \beta) \geq \mu_{2}>0, \quad x_{\beta}(\alpha, \beta) \geq \mu_{2}>0, \quad \forall(\alpha, \beta) \in \Pi^{-1}\left(\mathcal{O}_{\left(t_{0}, y\right)}^{\varepsilon_{*}}\right),
$$

where $\mu_{2}$ is a constant independent of $(\alpha, \beta)$. Using (1.3) and Lemma 3.2, we have

$$
\frac{\partial x_{+}(t, \alpha)}{\partial \alpha} \geq \mu_{3}>0, \quad \frac{\partial x_{-}(t, \beta)}{\partial \beta} \geq \mu_{3}>0, \quad \forall(\alpha, \beta) \in \Pi^{-1}\left(\mathcal{O}_{\left(t_{0}, y\right)}^{\varepsilon_{*}}\right)
$$

where $\mu_{3}$ is a constant independent of $(\alpha, \beta)$ and $t$. It is well known that $(3.23)$ implies (3.20). This completes the proof of Theorem 3.2.

Remark 3.2. By (2.4) and (3.20), we can extend the $C^{1}$ solution $(w(t, x), z(t, x))$ to a small neighbourhood of $\left(t_{0}, y\right)$, where $\left(t_{0}, y\right) \notin \mathcal{S}$. This means that the $C^{1}$ solution does not blow up at any point $\left(t_{0}, y\right) \notin \mathcal{S}$. 
We remark here that, in general, the fast (resp. slow) characteristics passing through a blow-up point are not unique. However, if we add some suitable assumptions, then we can get the uniqueness of such characteristics.

Theorem 3.3. If $w_{x}(t, x)$ (resp. $z_{x}(t, x)$ ) is uniformly bounded in the domain $D\left(t_{0}\right)$, then there exists a unique fast (resp. slow) characteristic passing through any point $(t, x) \in \overline{D\left(t_{0}\right)}$. In particular, if $w_{x}(t, x)$ (resp. $\left.z_{x}(t, x)\right)$ is uniformly bounded in $\mathcal{N}_{\left(t_{0}, x_{*}\right)} \cap D\left(t_{0}\right)$, then there exists a unique fast (resp. slow) characteristic passing through $\left(t_{0}, x_{*}\right)$, where $x_{*} \in \mathbb{R}$ and $\mathcal{N}_{\left(t_{0}, x_{*}\right)}$ is a neighbourhood of $\left(t_{0}, x_{*}\right)$.

Theorem 3.3 can be easily proved by contradiction, but we leave this to the reader.

\section{BLOW-UP OF CUSP TYPE}

In this section, under appropriate assumptions we prove that the solution of the Cauchy problem (1.1)-(1.2) is a blow-up solution of cusp type. Then, based on this, we construct the envelope of characteristics of the same family.

Suppose that $\lambda_{ \pm}(w, z) \in C^{4}$ and $w_{0}(x), z_{0}(x) \in C^{4}$. Suppose furthermore that there exists a point $\left(\alpha_{0}, \beta_{0}\right) \in J^{-1}(0) \cap t^{-1}\left(t_{0}\right)$ such that

$$
\begin{gathered}
x_{\alpha}\left(\alpha_{0}, \beta_{0}\right)>0, \quad x_{\beta}\left(\alpha_{0}, \beta_{0}\right)=0, \\
x_{\beta \beta}\left(\alpha_{0}, \beta_{0}\right)=0, \quad x_{\beta \beta \beta}\left(\alpha_{0}, \beta_{0}\right)>0 .
\end{gathered}
$$

Remark 4.1. By (1.3) and (2.7), (4.1) and (4.2) are respectively equivalent to

$$
\begin{aligned}
& t_{\alpha}\left(\alpha_{0}, \beta_{0}\right)<0, \quad t_{\beta}\left(\alpha_{0}, \beta_{0}\right)=0, \\
& t_{\beta \beta}\left(\alpha_{0}, \beta_{0}\right)=0, \quad t_{\beta \beta \beta}\left(\alpha_{0}, \beta_{0}\right)>0 .
\end{aligned}
$$

Remark 4.2. The hypothesis (4.1) implies that $z_{x}(t, x)$ blows up at $\left(t_{0}, x_{0}\right)$, but $w_{x}(t, x)$ does not blow up. We will discuss the case when two Riemann invariants blow up simultaneously at a point sometime in the future. In this case we believe that two shocks will grow out of the blow-up point if system (1.1) is of a form of conservation laws.

When the initial data (1.2) is of simple wave type, the hypotheses (4.1)-(4.2) can be expressed explicitly in terms of the initial data.

Proposition 4.1. If $w_{0}(x) \equiv \bar{w}_{0}=$ constant, then (4.1)-(4.2) are equivalent to $g^{\prime}(\beta)$ taking its global minimum at $\beta_{0}$ with

$$
g^{\prime}\left(\beta_{0}\right)<0, \quad g^{\prime \prime}\left(\beta_{0}\right)=0 \quad \text { and } \quad g^{\prime \prime \prime}\left(\beta_{0}\right)>0
$$

where $g(\beta)=\lambda_{-}\left(\bar{w}_{0}, z_{0}(\beta)\right)$.

Proof. Without loss of generality, we may suppose that $\bar{w}_{0} \equiv 0$. Hence, on the existence domain of a classical solution of the Cauchy problem (1.1)-(1.2), we have $w(t, x) \equiv 0$. Moreover, the slow characteristic passing through any fixed point $(0, \beta)$ is a straight line:

$$
x=\beta+g(\beta) t,
$$

where $g(\beta)=\lambda_{-}\left(0, z_{0}(\beta)\right)$; moreover, on $(4.3)$ we have $z=z_{0}(\beta)$.

Differentiating (4.3) with respect to $\beta$ twice gives

$$
x_{\beta \beta}=g^{\prime \prime}(\beta) t+2 g^{\prime}(\beta) t_{\beta}+g(\beta) t_{\beta \beta},
$$


and then differentiating the second equation in (2.7) with respect to $\beta$ yields

$$
x_{\beta \beta}=\frac{\partial \lambda_{+}}{\partial z}\left(0, z_{0}(\beta)\right) z_{0}^{\prime}(\beta) t_{\beta}+\lambda_{+}\left(0, z_{0}(\beta)\right) t_{\beta \beta} .
$$

Substituting (4.5) into (4.4) leads to

$$
\left[1-\frac{\lambda_{-}}{\lambda_{+}}\right] x_{\beta \beta}=g^{\prime \prime}(\beta) t+\left[2 g^{\prime}(\beta)-\frac{\lambda_{-}}{\lambda_{+}} \frac{\partial \lambda_{+}}{\partial z} z_{0}^{\prime}(\beta)\right] t_{\beta},
$$

where $\lambda_{ \pm}=\lambda_{ \pm}\left(0, z_{0}(\beta)\right)$.

Step I: (4.1)-(4.2) $\Longrightarrow(\mathrm{H})$. By Theorem 3.2, the assumption that $x_{\beta}\left(\alpha_{0}, \beta_{0}\right)=$ 0 implies that $z_{x}(t, x)$ must blow up at $\left(t_{0}, x_{0}\right)$, but it does not blow up for any $t<t_{0}$. Noting (4.3), we see that $g^{\prime}(\beta)$ must take its global minimum at $\beta_{0}$. Moreover, combining (1.4) and (3.15) gives $g^{\prime}\left(\beta_{0}\right)<0$ immediately.

We now prove $g^{\prime \prime}\left(\beta_{0}\right)=0$ and $g^{\prime \prime \prime}\left(\beta_{0}\right)>0$.

Using (4.1)-(4.2) and noting that $t_{0}=t\left(\alpha_{0}, \beta_{0}\right)>0$, we obtain from (4.6) that $g^{\prime \prime}\left(\beta_{0}\right)=0$.

Similarly, we can prove that $g^{\prime \prime \prime}\left(\beta_{0}\right)>0$.

Step II: $(\mathrm{H}) \Longrightarrow(4.1)-(4.2)$.

Since $w \equiv 0$, we observe that the Cauchy problem (1.1)-(1.2) can be reduced to

$$
\partial_{t} z+\lambda_{-}(0, z) \partial_{x} z=0, \quad z(0, x)=z_{0}(x)
$$

on the existence domain of a classical solution. Since $g^{\prime}(\beta)$ takes its global minimum at $\beta_{0}$ and $g^{\prime}(\beta)<0$, by the classical theory for a single equation, we have

$$
\begin{aligned}
& \frac{\partial x_{-}(t, \beta)}{\partial \beta}>0, \quad \forall t \in\left[0, t_{0}\right), \quad \forall \beta \in \mathbb{R}, \\
& \left.\frac{\partial x_{-}(t, \beta)}{\partial \beta}\right|_{\beta=\beta_{0}} \longrightarrow 0 \quad \text { as } t \nearrow t_{0},
\end{aligned}
$$

where $t_{0}=-\left(g^{\prime}\left(\beta_{0}\right)\right)^{-1}$ and $x_{-}(t, \beta)=\beta+\lambda_{-}\left(0, z_{0}(\beta)\right) t$. Thus, $z_{x}=z_{x}(t, x)$ blows up at $\left(t_{0}, x_{0}\right) \triangleq\left(t_{0}, \beta_{0}+g\left(\beta_{0}\right) t_{0}\right)$, but it does not blow up for any $t<t_{0}$.

Still consider the Cauchy problem (1.1)-(1.2). Since $w \equiv 0$, by Theorem 3.3 we can draw a unique fast characteristic passing through the point $\left(t_{0}, x_{0}\right)$. Choose the characteristic coordinates as in $\S 2$ and take the $x$-coordinate of the intersection point of the fast characteristic passing through $\left(t_{0}, x_{0}\right)$ with the $x$-axis as $\alpha_{0}$. Noting (4.7) and using (3.9), we get that $x_{\beta}\left(\alpha_{0}, \beta_{0}\right)=0$. On the other hand, since $w$ does not blow up, we know that $x_{\alpha}\left(\alpha_{0}, \beta_{0}\right)>0$. Moreover, noting that $g^{\prime \prime}\left(\beta_{0}\right)=0$ and using (1.3), we obtain from (4.6) that $x_{\beta \beta}\left(\alpha_{0}, \beta_{0}\right)=0$.

Similarly, using the fact that $g^{\prime \prime \prime}\left(\beta_{0}\right)>0$, we can prove that $x_{\beta \beta \beta}\left(\alpha_{0}, \beta_{0}\right)>0$. This proves (4.1)-(4.2).

Remark 4.3. For the $p$-system, $(\mathrm{H})$ is nothing but the assumption used in [13].

By (4.1)-(4.2), there exists a full neighbourhood $\mathcal{O}$ of $\left(\alpha_{0}, \beta_{0}\right)$ such that

$$
\left.x_{\alpha}(\alpha, \beta)>0 \text { (equiv. } t_{\alpha}(\alpha, \beta)<0\right), \quad x_{\beta \beta \beta}(\alpha, \beta)>0, \quad \forall(\alpha, \beta) \in \mathcal{O} .
$$

Let $\mathcal{O}_{0}$ be a small ball centered at $\left(\alpha_{0}, \beta_{0}\right)$ and satisfying $\mathcal{O}_{0} \subseteq \mathcal{O}$. In what follows, we investigate the behaviour and structure of the set $J^{-1}(0) \cap \mathcal{O}_{0}$, i.e., the set $C_{z} \triangleq\left\{(\alpha, \beta) \mid(\alpha, \beta) \in \mathcal{O}_{0}, x_{\beta}(\alpha, \beta)=0\right\}$. 
Theorem 4.1. Suppose that (1.3)-(1.4) and (4.1)-(4.2) hold. Then in $\mathcal{O}_{0}$, the singular points of $\Pi$ form a smooth curve which can be defined by an explicit function $\alpha=\alpha(\beta)$ with the following properties:

(1) $\alpha\left(\beta_{0}\right)=\alpha_{0}$;

(2) $\alpha=\alpha(\beta)$ is strictly concave, and takes its maximum at $\beta_{0}$;

(3) singular points $(\alpha, \beta)\left(\neq\left(\alpha_{0}, \beta_{0}\right)\right)$ are fold points, and $\left(\alpha_{0}, \beta_{0}\right)$ is a cusp point.

Proof. Differentiating (2.9) with respect to $\alpha$ gives

$$
J_{\alpha}=\left(\frac{1}{\lambda_{-}}-\frac{1}{\lambda_{+}}\right) x_{\alpha}\left(x_{\beta}\right)_{\alpha} \quad \text { on } \quad C_{z} .
$$

Moreover, differentiating the first equation in (2.7) with respect to $\beta$ yields

$$
\left(1-\frac{\lambda_{-}}{\lambda_{+}}\right)\left(x_{\beta}\right)_{\alpha}=\frac{\partial \lambda_{-}}{\partial z} z_{0}^{\prime}(\beta) t_{\alpha} \quad \text { on } \quad C_{z} .
$$

Noting (1.3)-(1.4) and using (3.15) and (4.8), from (4.10) we get

$$
\left(x_{\beta}\right)_{\alpha}>0 \text { on } C_{z} \text {. }
$$

Then, noting (1.3) and (4.8), we obtain from (4.9) that

$$
J_{\alpha}<0 \text { on } C_{z} .
$$

This implies that the mapping $\Pi$ is good in $\mathcal{O}_{0}$. Therefore, the singular points of $\Pi$ define a smooth curve in $\mathcal{O}_{0}$ (see [20]). Let us parametrize the curve $J(\alpha, \beta)=0$ by $\Gamma(\sigma)=(\alpha(\sigma), \beta(\sigma))$. By (4.12), the curve can be expressed by an explicit function denoted by $\alpha=\alpha(\beta)$ with $\alpha\left(\beta_{0}\right)=\alpha_{0}$. Obviously,

$$
\left.x_{\beta}(\alpha(\beta), \beta)=0 \quad \text { (equivalently, } t_{\beta}(\alpha(\beta), \beta)=0\right) .
$$

Differentiating (4.13) with respect to $\beta$ and noting (4.2) and (4.11), we obtain

$$
\alpha^{\prime}\left(\beta_{0}\right)=0 \quad \text { and } \quad \alpha^{\prime \prime}\left(\beta_{0}\right)<0 .
$$

This shows that $\alpha=\alpha(\beta)$ is strictly concave in $\mathcal{O}_{0}$ and takes its maximum at $\beta_{0}$, provided that the ball $\mathcal{O}_{0}$ is suitably small.

By a direct calculation, we have

$$
\begin{aligned}
& \frac{d}{d \beta}(t(\alpha(\beta), \beta), x(\alpha(\beta), \beta)) \neq(0,0), \quad \forall \beta \neq \beta_{0}, \\
& \left.\frac{d}{d \beta}(t(\alpha(\beta), \beta), x(\alpha(\beta), \beta))\right|_{\beta=\beta_{0}}=(0,0), \\
& \left.\frac{d^{2}}{d \beta^{2}}(t(\alpha(\beta), \beta), x(\alpha(\beta), \beta))\right|_{\beta=\beta_{0}} \neq(0,0) .
\end{aligned}
$$

(4.15) implies that $(\alpha(\beta), \beta)\left(\beta \neq \beta_{0}\right)$ is a fold point of the mapping $\Pi$, while $\left(\alpha_{0}, \beta_{0}\right)$ is a cusp point. This proves the theorem.

Remark 4.4. Theorem 4.1 shows that under the hypotheses (1.3)-(1.4) and (4.1)(4.2), the solution of the Cauchy problem (1.1)-(1.2) is a blow-up solution of cusp type, according to the terminology of [1]. Moreover, Theorem 4.1 can be viewed as an improvement of Theorem 3.2 in Chapter IV of [1]. 


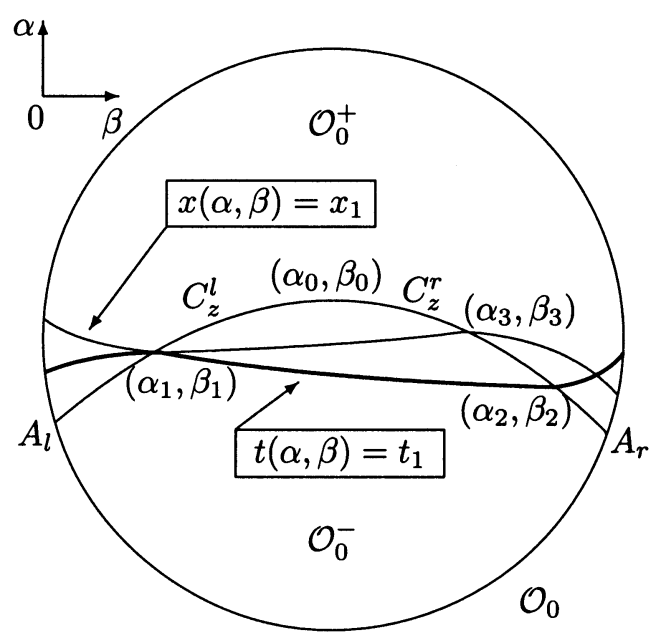

FiguRE 1. Smooth curve $C_{z}=C_{z}^{l} \cup C_{z}^{r}$ formed by singular points of mapping $\Pi$

From results of Whitney [20] and Theorem 3.3, as two corollaries of Theorem 4.1 we have

Corollary 4.1. Suppose that $\lambda_{ \pm}(w, z)$ are $C^{\infty}$ functions of $(w, z)$, and $w_{0}(x)$ and $z_{0}(x)$ are $C^{\infty}$ functions of $x$ with bounded $C^{1}$ norm. Suppose furthermore that (1.3)-(1.4) and (4.1)-(4.2) hold. Then there exist smooth coordinate systems $(\bar{\alpha}, \bar{\beta})$ and $(\bar{t}, \bar{x})$, near $\left(\alpha_{0}, \beta_{0}\right)$ and $\left(t_{0}, x_{0}\right)$ respectively, such that the mapping $\Pi$ takes the form $\bar{x}=\bar{\alpha} \bar{\beta}-\bar{\beta}^{3}, \quad \bar{t}=\bar{\alpha}$.

Corollary 4.2. Under the hypotheses (1.3)-(1.4) and (4.1)-(4.2), there exists a unique fast (resp. slow) characteristic passing through $\left(t_{0}, x_{0}\right) \in \mathcal{S}$.

Let $C_{z}^{l}$ (resp. $C_{z}^{r}$ ) be the part of the curve $\alpha=\alpha(\beta)$ to the left (resp. right) of $\left(\alpha_{0}, \beta_{0}\right)$ in $\mathcal{O}_{0}$ (see Fig. 1). Obviously, $C_{z}=C_{z}^{l} \cup C_{z}^{r}$, and $C_{z}^{l}$ (resp. $C_{z}^{r}$ ) is the strictly increasing (resp. decreasing) part. Furthermore, let $\gamma^{l}$ (resp. $\gamma^{r}$ ) be the image of $C_{z}^{l}$ (resp. $C_{z}^{r}$ ) under the mapping $\Pi$. In what follows, we consider the behaviour of $\gamma^{l}$ and $\gamma^{r}$ in the $(t, x)$-plane.

To do so, we introduce

$$
\begin{aligned}
& \mathcal{O}_{0}^{+}=\left\{(\alpha, \beta) \mid(\alpha, \beta) \in \mathcal{O}_{0}, \alpha>\alpha(\beta)\right\}, \\
& \mathcal{O}_{0}^{-}=\left\{(\alpha, \beta) \mid(\alpha, \beta) \in \mathcal{O}_{0}, \alpha<\alpha(\beta)\right\},
\end{aligned}
$$

and denote the intersection points of the curve $\alpha=\alpha(\beta)$ with the boundary of $\mathcal{O}_{0}$ by $A_{l}\left(\alpha\left(\beta_{l}\right), \beta_{l}\right)$ and $A_{r}\left(\alpha\left(\beta_{r}\right), \beta_{r}\right)$ respectively (see Fig. 1).

Lemma 4.1. We have

$$
\begin{array}{lll}
t_{\alpha}(\alpha, \beta)<0 & \text { (equivalently, } \left.x_{\alpha}(\alpha, \beta)>0\right) \quad \text { in } \quad \mathcal{O}_{0}, \\
t_{\beta}(\alpha, \beta)>0 & \text { (equivalently, } \left.x_{\beta}(\alpha, \beta)>0\right) \quad \text { in } \quad \mathcal{O}_{0}^{+}, \\
t_{\beta}(\alpha, \beta)<0 & \text { (equivalently, } \left.x_{\beta}(\alpha, \beta)<0\right) \quad \text { in } \quad \mathcal{O}_{0}^{-} .
\end{array}
$$

Proof. (4.17) $)_{1}$ comes from (4.8) directly. It remains to prove $(4.17)_{2}-(4.17)_{3}$. 
By (2.7), we can easily get

$$
\left(t_{\beta}\right)_{\alpha}(\alpha, \beta)=\frac{1}{\lambda_{-}-\lambda_{+}} \frac{\partial \lambda_{+}}{\partial w} w_{0}^{\prime}(\alpha) t_{\beta}+\frac{1}{\lambda_{+}-\lambda_{-}} \frac{\partial \lambda_{-}}{\partial z} z_{0}^{\prime}(\beta) t_{\alpha} .
$$

Moreover, from (4.13) we have

$$
t_{\beta}(\alpha(\beta), \beta)=0, \quad \forall \beta \in\left[\beta_{l}, \beta_{r}\right] .
$$

For any fixed $\beta \in\left[\beta_{l}, \beta_{r}\right]$, we solve the initial value problem (4.18)-(4.19) and obtain

$$
t_{\beta}(\alpha, \beta)=\int_{\alpha(\beta)}^{\alpha} G(\zeta, \beta) \exp Q(\zeta, \alpha, \beta) d \zeta, \quad \forall(\alpha, \beta) \in \mathcal{O}_{0},
$$

where

$$
Q(\zeta, \alpha, \beta) \triangleq \int_{\zeta}^{\alpha} \frac{1}{\lambda_{-}\left(w_{0}(\xi), z_{0}(\beta)\right)-\lambda_{+}\left(w_{0}(\xi), z_{0}(\beta)\right)} \frac{\partial \lambda_{+}}{\partial w}\left(w_{0}(\xi), z_{0}(\beta)\right) w_{0}^{\prime}(\xi) d \xi
$$

and

$$
G(\alpha, \beta) \triangleq \frac{1}{\lambda_{+}-\lambda_{-}} \frac{\partial \lambda_{-}}{\partial z} z_{0}^{\prime}(\beta) t_{\alpha}
$$

Noting (1.3)-(1.4), (3.15) and (4.17) $)_{1}$, we observe that $G(\alpha, \beta)>0, \forall(\alpha, \beta) \in \mathcal{O}_{0}$. Hence, from $(4.20)$ we obtain $(4.17)_{2}-(4.17)_{3}$ immediately. The proof is complete.

Moreover, it follows from $(4.17)_{1}$ that, in $\mathcal{O}_{0}, t(\alpha, \beta)=$ const. (resp. $x(\alpha, \beta)=$ const.) can be explicitly expressed by $\alpha=\alpha^{t}(\beta) \quad\left(\right.$ resp. $\left.\alpha=\alpha^{x}(\beta)\right)$. Using Lemma 4.1 , we can easily prove

Lemma 4.2. The curve $t(\alpha, \beta)=$ const. is increasing in $\mathcal{O}_{0}^{+}$and decreasing in $\mathcal{O}_{0}^{-}$, while the curve $x(\alpha, \beta)=$ const. is decreasing in $\mathcal{O}_{0}^{+}$and increasing in $\mathcal{O}_{0}^{-}$.

Since $\gamma^{l}$ (resp. $\gamma^{r}$ ) is the image of $C_{z}^{l}$ (resp. $C_{z}^{r}$ ) under the mapping $\Pi$, it is easy to see that $\gamma^{l}$ (resp. $\gamma^{r}$ ) is defined by

$$
x=x(\alpha(\beta), \beta), \quad t=t(\alpha(\beta), \beta), \quad \forall \beta \in\left[\beta_{l}, \beta_{0}\right] \quad\left(\text { resp. } \forall \beta \in\left[\beta_{0}, \beta_{r}\right]\right)
$$

in particular, $x_{0}=x\left(\alpha\left(\beta_{0}\right), \beta_{0}\right), t_{0}=t\left(\alpha\left(\beta_{0}\right), \beta_{0}\right)$. Noting (4.14), (4.17) 1 and differentiating $(4.21)$ with respect to $\beta$, we obtain

$$
\begin{aligned}
& \frac{d x}{d \beta}>0 \quad \text { and } \quad \frac{d t}{d \beta}<0, \quad \forall \beta \in\left(\beta_{l}, \beta_{0}\right), \\
& \frac{d x}{d \beta}<0 \quad \text { and } \quad \frac{d t}{d \beta}>0, \quad \forall \beta \in\left(\beta_{0}, \beta_{r}\right) .
\end{aligned}
$$

(4.22) implies that $\gamma^{l}$ and $\gamma^{r}$ are strictly decreasing in the $(t, x)$-plane. Let $P_{0}$ be $\left(t_{0}, x_{0}\right)$. Obviously, the lowest and rightmost point of $\gamma^{l}$ and $\gamma^{r}$ is just $P_{0}$.

On the other hand, noting (4.22) and using the implicit function theorem, we can solve for $\beta$ from $t=t(\alpha(\beta), \beta)$ when $\beta \in\left(\beta_{l}, \beta_{0}\right)$ (resp. $\left.\beta \in\left(\beta_{0}, \beta_{r}\right)\right)$, and denote it by $\beta=\beta^{l}(t)$ (resp. $\beta=\beta^{r}(t)$ ), where $t \in\left(t_{0}, t\left(\alpha\left(\beta_{l}\right), \beta_{l}\right)\right.$ ) (resp. $\left.t \in\left(t_{0}, t\left(\alpha\left(\beta_{r}\right), \beta_{r}\right)\right)\right)$. Substituting it into $x=x(\alpha(\beta), \beta)$, we get an explicit 


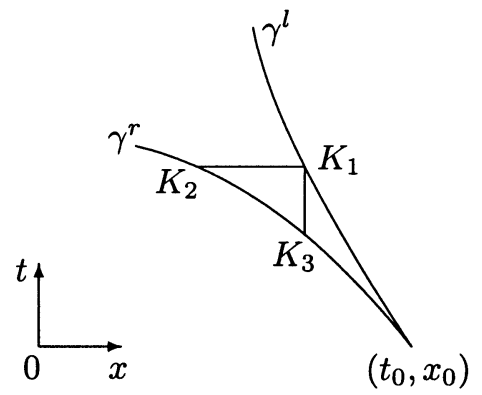

Figure 2. Envelope of slow characteristics

formula for $\gamma^{l}$ (resp. $\gamma^{r}$ ), and denote it by $x=x_{l}(t)$ (resp. $x=x_{r}(t)$ ), where $t \in\left(t_{0}, t\left(\alpha\left(\beta_{l}\right), \beta_{l}\right)\right)$ (resp. $\left.t \in\left(t_{0}, t\left(\alpha\left(\beta_{r}\right), \beta_{r}\right)\right)\right)$. By a direct calculation, we have

$$
\begin{aligned}
& \frac{d^{2} x_{l}(t)}{d t^{2}}>0, \quad \forall t \in\left(t_{0}, t\left(\alpha\left(\beta_{l}\right), \beta_{l}\right)\right), \\
& \frac{d^{2} x_{r}(t)}{d t^{2}}<0, \quad \forall t \in\left(t_{0}, t\left(\alpha\left(\beta_{r}\right), \beta_{r}\right)\right) .
\end{aligned}
$$

(4.23) implies that $\gamma^{l}$ is strictly convex and $\gamma^{r}$ is strictly concave.

Choose a point $\left(\alpha_{1}, \beta_{1}\right)$ on $C_{z}^{l}$, and let $x_{1}$ be $x\left(\alpha_{1}, \beta_{1}\right)$ and $t_{1}$ be $t\left(\alpha_{1}, \beta_{1}\right)$. We draw two curves: $t(\alpha, \beta)=t_{1}$ and $x(\alpha, \beta)=x_{1}$ passing through $\left(\alpha_{1}, \beta_{1}\right)$. Let $\left(\alpha_{j}, \beta_{j}\right)(j=2,3)$ be the intersection points of $C_{z}^{r}$ with $t(\alpha, \beta)=t_{1}$ and $x(\alpha, \beta)=$ $x_{1}$ respectively (see Fig. 1 ). As in Fig. 1 , we see that $\beta_{2}>\beta_{3}$. Then using $(4.22)_{2}$, we have

$$
\begin{aligned}
& x_{1}=x_{3} \triangleq x\left(\alpha_{3}, \beta_{3}\right)>x_{2} \triangleq x\left(\alpha_{2}, \beta_{2}\right), \\
& t_{3} \triangleq t\left(\alpha_{3}, \beta_{3}\right)<t_{2} \triangleq t\left(\alpha_{2}, \beta_{2}\right)=t_{1} .
\end{aligned}
$$

(4.24) implies that $\gamma^{r}$ lies below $\gamma^{l}$, where $K_{i}=\left(t\left(\alpha_{i}, \beta_{i}\right), x\left(\alpha_{i}, \beta_{i}\right)\right)(i=1,2,3)$. See Fig. 2. Summarizing the above discussion, we have

Theorem 4.2. Under the assumptions (1.3)-(1.4) and (4.1)-(4.2), $\gamma^{l}$ and $\gamma^{r}$ are strictly decreasing in the $(t, x)$-plane, and $\left(t_{0}, x_{0}\right)$ is the lowest and rightmost point of $\gamma^{l}$ and $\gamma^{r}$; moreover, $\gamma^{l}$ is strictly concave, while $\gamma^{r}$ is strictly convex and lies below $\gamma^{l}$.

Remark 4.5. $\gamma^{l}$ and $\gamma^{r}$ can be regarded as two sheets of the envelope with its cusp at $\left(t_{0}, x_{0}\right)$ formed by slow characteristics. Moreover, Theorem 4.2 shows that under the hypotheses (1.3)-(1.4) and (4.1)-(4.2), the formation of a singularity of the classical solution of the Cauchy problem (1.1)-(1.2) is due to the envelope of slow characteristics and the singularity occurs at the starting point of the envelope, i.e., the point with minimum $t$-value on the envelope.

In what follows we introduce the triple-valued solution and the artificial solution of the Cauchy problem (1.1)-(1.2) in a neighbourhood of $\left(t_{0}, x_{0}\right)$.

Let $R_{0}$ be the point $\left(\alpha_{0}, \beta_{0}\right)$. Choose a point $R_{3} \in C_{z}^{r}$, and a point $R_{1}$ to the right side of $R_{3}$ in $\mathcal{O}_{0}$ and on the line $\alpha=\alpha_{0}$, such that the intersection point $R_{2}$ of the vertical line passing through $R_{1}$ and the horizontal line passing through $R_{3}$ also lies in $\mathcal{O}_{0}$. Let $D_{+}$be the region bounded by $C_{z}^{r}, \alpha=\alpha_{0}$, the vertical segment 


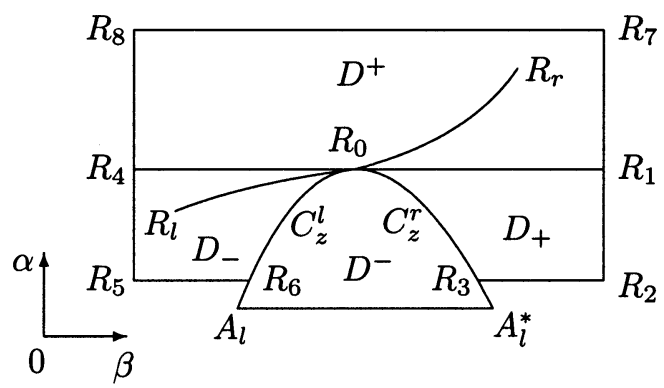

Figure 3. Points $R_{i}(i=0,1, \ldots, 8, l, r)$ in $(\alpha, \beta)$-plane

$R_{1} R_{2}$ and the horizontal segment $R_{3} R_{2}$. $D_{-}$can be defined in a similar way (see Fig. 3). Moreover, let $D^{-}=\left\{(\alpha, \beta) \mid(\alpha, \beta) \in \mathcal{O}_{0}^{-}, \max \left(\alpha\left(\beta_{l}\right), \alpha\left(\beta_{r}\right)\right) \leq \alpha \leq \alpha_{0}\right\}$. In Fig. 3 we have assumed that $\alpha\left(\beta_{l}\right) \geq \alpha\left(\beta_{r}\right)$ and have denoted the intersection point of $C_{z}^{r}$ with the horizontal line passing through point $A_{l}$ by $A_{l}^{*}$. Finally, choose a point $R_{7}$ (resp. $R_{8}$ ) to the upside of $R_{1}$ (resp. $R_{4}$ ) and on the vertical line passing through $R_{1}$ (resp. $R_{4}$ ), such that the region $R_{1} R_{7} R_{8} R_{4}$, denoted by $D^{+}$, is still in $\mathcal{O}_{0}$ (see Fig. 3).

Let $\Pi=\Pi^{ \pm}(\alpha, \beta)$ and $\Pi=\Pi_{ \pm}(\alpha, \beta)$ be the restrictions of the solution $(t, x)=$ $(t(\alpha, \beta), x(\alpha, \beta))$ of Cauchy problem (2.7)-(2.8) to $D^{ \pm}$and $D_{ \pm}$respectively, and let $P_{i}$ be $\Pi\left(R_{i}\right)(i=1, \cdots, 8)$ and $P^{+}, P^{-}$be $\Pi\left(A_{l}^{*}\right), \Pi\left(A_{l}\right)$ respectively (see Fig. 4). In determining the positions of $P_{i}$ in the $(t, x)$-plane, we use the fact that the image of $\alpha=$ const. is described by $\frac{d x}{d t}=\lambda_{+}\left(w_{0}(\alpha), z_{0}(\beta)\right)>0$ and then is an increasing curve in the $(t, x)$-plane. Similarly, the image of $\beta=$ const. is a decreasing curve. It easy to see that $P_{0} P_{1} P_{2} P_{3}=\Pi_{+}\left(D_{+}\right), P_{0} P_{4} P_{5} P_{6}=\Pi_{-}\left(D_{-}\right), P_{0} P^{+} P^{-}=\Pi^{-}\left(D^{-}\right)$ and $P_{1} P_{4} P_{8} P_{7}=\Pi^{+}\left(D^{+}\right)$. Since the inverse mappings of $\Pi=\Pi^{ \pm}(\alpha, \beta)$ and $\Pi=$ $\Pi_{ \pm}(\alpha, \beta)$ exist on their corresponding domains, we may construct the $C^{4}$ solution of system (1.1) in the regions $\Pi^{ \pm}\left(D^{ \pm}\right)$and $\Pi_{ \pm}\left(D_{ \pm}\right)$respectively, denoted by $(w, z)=$ $\left(w^{ \pm}(t, x), z^{ \pm}(t, x)\right)$ and $(w, z)=\left(w_{ \pm}(t, x), z_{ \pm}(t, x)\right)$ respectively. Moreover, it is easy to show that $\left(w^{+}(t, x), z^{+}(t, x)\right)$ and $\left(w_{+}(t, x), z_{+}(t, x)\right)$ can be extended to the curve $P_{0} P_{1}$ except for the point $P_{0}$ and connected to each other with $C^{4}$ regularity. $\left(w^{+}(t, x), z^{+}(t, x)\right)$ and $\left(w_{-}(t, x), z_{-}(t, x)\right)$ have a similar property on the curve $P_{0} P_{4}$ except $P_{0}$. As in Fig. 4, let $\Omega_{0}$ be the open region bounded by $\gamma^{r}, \gamma^{l}$ and the curve $P_{2} P_{3}$. Clearly, the "solution" constructed in the previous way is triple-valued in $\Omega_{0}$. Such a "solution" is called the triple-valued solution of the Cauchy problem (1.1)-(1.2). However, we cannot construct a shock growing out of the point $\left(t_{0}, x_{0}\right)$ by solving the initial value problem for a nonlinear ODE as in the case of a single conservation law, since if we ask this ODE to be given by one of the RankineHugoniot conditions with $\left(w_{ \pm}(t, x), z_{ \pm}(t, x)\right)$ as the values on both sides, another one of the Rankine-Hugoniot conditions is not satisfied generally. In $\S 5$ we will discuss this problem in a different way. However, here we can construct at least one artificial solution $(w, z)$ in a neighbourhood of $\left(t_{0}, x_{0}\right)$ in the following way, gluing together the piecewise solutions:

(1) $(w, z)=\left(w^{+}(t, x), z^{+}(t, x)\right)$ in the region $P_{1} P_{4} P_{8} P_{7} \backslash\left\{P_{0}\right\}$;

(2) $(w, z)=\left(w_{-}(t, x), z_{-}(t, x)\right)$ in the region $\Omega_{-} \backslash\left\{P_{0}\right\}$, where $\Omega_{-}$is the region bounded by the curves $P_{0} P_{4}, P_{4} P_{5}, P_{5} P_{6}$ and $P_{0} P_{*}$, in which $P_{0} P_{*}$ is an 


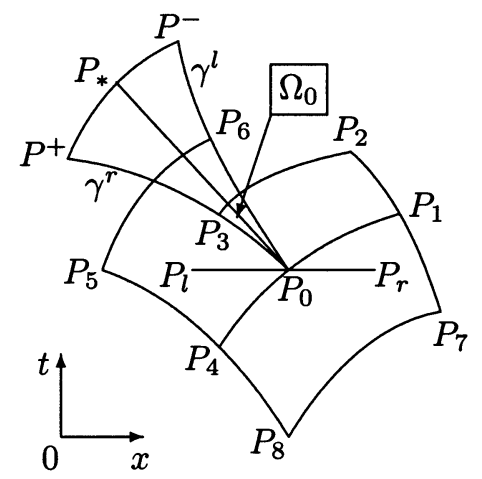

FiguRE 4. Images of $R_{i}$ in $(t, x)$-plane

arbitrary strictly decreasing $C^{1}$ curve in the region $P^{+} P_{0} P^{-}$and satisfies $P_{0} P_{*} \cap P_{0} P^{-}=P_{0} P_{*} \cap P_{0} P^{+}=\left\{P_{0}\right\}$ (see Fig. 4);

(3) $(w, z)=\left(w_{+}(t, x), z_{+}(t, x)\right)$ in the region $\Omega_{+} \backslash\left\{P_{0}\right\}$, where $\Omega_{+}$is the region bounded by the curves $P_{0} P_{1}, P_{1} P_{2}, P_{2} P_{3}$ and $P_{0} P_{*}$.

Obviously, such an artificial solution is a piecewise $C^{4}$ function which satisfies the system (1.1) in a neighbourhood of $\left(t_{0}, x_{0}\right)$ except for the curve $P_{0} P_{*}$. Moreover, when $t<t_{0}$ or $x>x_{+}\left(t, \alpha_{0}\right)$, the artificial solution is nothing but the $C^{4}$ solution of the Cauchy problem (1.1)-(1.2). Thus we have

Theorem 4.3. Under the hypotheses (1.3)-(1.4) and (4.1)-(4.2), the Cauchy problem (1.1)-(1.2) admits a triple-valued solution in a neighbourhood of $\left(t_{0}, x_{0}\right)$. Particularly, for any fixed $C^{1}$ smooth curve $P_{0} P_{*} \subseteq \Omega_{0}$ there exists an artificial solution of the Cauchy problem (1.1)-(1.2) in a neighbourhood of $\left(t_{0}, x_{0}\right)$.

To conclude this section, we state the following theorem, whose standard proof is left to the reader.

Theorem 4.4. Under the hypotheses (1.3)-(1.4) and (4.1)-(4.2), the solution $(w, z)$ of the Cauchy problem (1.1)-(1.2) satisfies the following estimates:

$$
\begin{aligned}
& \left|w(t, x)-w\left(t_{0}, x_{0}\right)\right| \leq k_{0}\left(\left|t-t_{0}\right|+\left|x-x_{0}\right|\right), \quad \forall(t, x) \in \mathcal{N}^{\#}, \\
& \left|w_{x}(t, x)-w_{x}\left(t_{0}, x_{0}\right)\right| \leq k_{0}\left(\left|t-t_{0}\right|+\left|x-x_{0}\right|\right), \quad \forall(t, x) \in \mathcal{N}^{\#}, \\
& \left|z(t, x)-z\left(t_{0}, x_{0}\right)\right| \leq k_{1}\left(\left|t-t_{0}\right|^{3}+\left|x-x_{0}(t)\right|^{2}\right)^{\frac{1}{6}}, \quad \forall(t, x) \in \mathcal{N}^{\#}, \\
& \left|z_{x}(t, x)\right| \leq k_{2}\left(\left|t-t_{0}\right|^{3}+\left|x-x_{0}(t)\right|^{2}\right)^{-\frac{1}{3}}, \quad \forall(t, x) \in \mathcal{N}^{\#} \backslash\left\{\left(t_{0}, x_{0}\right)\right\}, \\
& \left|z_{x x}(t, x)\right| \leq k_{3}\left(\left|t-t_{0}\right|^{3}+\left|x-x_{0}(t)\right|^{2}\right)^{-\frac{5}{6}}, \quad \forall(t, x) \in \mathcal{N}^{\#} \backslash\left\{\left(t_{0}, x_{0}\right)\right\},
\end{aligned}
$$

where the $k_{i}(i=0,1,2,3)$ are positive constants independent of $(t, x), x_{0}(t)$ stands for the slow characteristic passing through $\left(0, \beta_{0}\right)$, and $\mathcal{N}^{\#}$ denotes a small neighbourhood of $\left(t_{0}, x_{0}\right)$ with $t \leq t_{0}$. 


\section{FORMATION OF SHOCK}

Consider the following Cauchy problem for a system of $2 \times 2$ conservation laws:

$$
\begin{gathered}
\left\{\begin{array}{l}
\frac{\partial u}{\partial t}+\frac{\partial f(u, v)}{\partial x}=0, \\
\frac{\partial v}{\partial t}+\frac{\partial g(u, v)}{\partial x}=0,
\end{array}\right. \\
t=0: \quad u=u_{0}(x), v=v_{0}(x),
\end{gathered}
$$

where $u, v$ are the unknown functions, $f(u, v), g(u, v) \in C^{5}(D)$ for some open domain $D$ in $\mathbb{R}^{2}, u_{0}(x), v_{0}(x) \in C^{4}(\mathbb{R})$ and their $C^{1}$ norms are bounded.

Suppose the system (5.1) is strictly hyperbolic, that is, $\nabla(f, g)^{\mathrm{T}}$ has two real and distinct eigenvalues. As in $\S 1$, we assume that

$$
\lambda_{1}(u, v)<0<\lambda_{2}(u, v) .
$$

Suppose furthermore that system (5.1) is genuinely nonlinear in the sense of Lax; without loss of generality, we may assume that

$$
\nabla \lambda_{i}(u, v) \cdot r_{i}(u, v)>0 \quad(i=1,2),
$$

where $r_{i}(u, v)$ is the right eigenvector corresponding to $\lambda_{i}(u, v)$.

Introducing the Riemann invariant $5=w(u, v)$ and $z=z(u, v)$, we may rewrite (5.1) as a system with the form (1.1) on any existence domain of smooth solution, and rewrite (5.2) as a condition with the form (1.2). In the new system (1.1) $w, z$ are the new unknown functions and $\lambda_{ \pm}(w, z)$ are smooth functions of $(w, z)$ satisfying $\lambda_{-}(w(u, v), z(u, v))=\lambda_{1}(u, v)$ and $\lambda_{+}(w(u, v), z(u, v))=\lambda_{2}(u, v)$, while in (1.2) $w_{0}(x), z_{0}(x)$ are $C^{4}$ functions with bounded $C^{1}$ norm. Obviously, (5.3) and (5.4) are equivalent to (1.3) and (1.4) respectively, since $\lambda_{i}, \nabla \lambda_{i} \cdot r_{i}(i=1,2)$ are invariants under any invertible $C^{1}$ transformation of $(u, v)$.

As before, let $(t(\alpha, \beta), x(\alpha, \beta))$ be the solution of the Cauchy problem (2.7)-(2.8), $\left(t_{0}, x_{0}\right)$ an arbitrary blow-up point in $\mathcal{S}$ and $\left(\alpha_{0}, \beta_{0}\right)$ a preimage of $\left(t_{0}, x_{0}\right)$. Suppose furthermore that (4.1)-(4.2) hold.

It is well known that the following problems are very interesting but quite open: What kinds of singularities will appear at the blow-up point $\left(t_{0}, x_{0}\right) \in \mathcal{S}$ ? How do the singularities (in particular, shocks) grow out of nothing? In this section, under the hypotheses (5.3)-(5.4) and (4.1)-(4.2) we study these problems. The main result is the following.

Theorem 5.1. Under the hypotheses (5.3)-(5.4) and (4.1)-(4.2), the Cauchy problem (5.1)-(5.2) has a unique classical discontinuous solution $(u(t, x), v(t, x))$ in a neighbourhood of the blow-up point $\left(t_{0}, x_{0}\right)$ : the solution contains a shock $x=$ $\varphi(t)\left(t \geq t_{0}\right)$ and a weak discontinuity $x=\psi(t)\left(t \geq t_{0}\right)$ issuing from $\left(t_{0}, x_{0}\right)$, and $(u(t, x), v(t, x))$ is $C^{4}$ smooth away from the curves $x=\varphi(t)$ and $x=\psi(t)$. See Fig. 5.

Remark 5.1. The weak discontinuity mentioned above means that the solution is continuous across $x=\psi(t)$, but its derivatives can be discontinuous. The weak discontinuity must propagate along the corresponding characteristic.

\footnotetext{
${ }^{5}$ As in Remark 1.1, the Riemann invariants always exist at least in a local domain.
} 


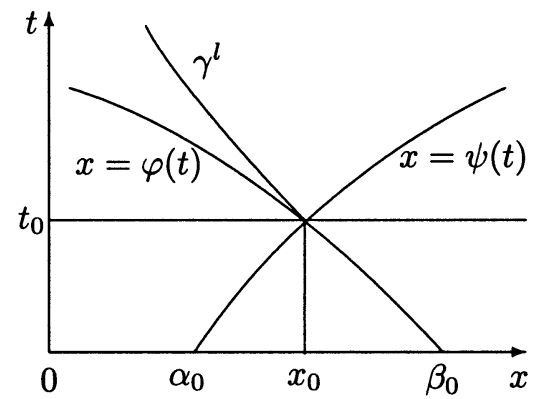

FIGURE 5. Shock and weak discontinuity issuing from $\left(t_{0}, x_{0}\right)$

Remark 5.2. By Proposition 4.1, if (5.1) is the $p$-system, then Theorem 5.1 gives Lebaud's result on the existence of a classical discontinuous solution in a neighbourhood of a blow-up point (see [13]). However, we do not need the assumption in 13 that one of the Riemann invariants is a constant.

In order to prove Theorem 5.1, it suffices to solve a kind of free boundary problem with one characteristic boundary on an angular domain. The difficulty of this kind of free boundary problem is that one boundary condition is not Lipschitz continuous at the angular point. The key idea to overcome the difficulty is as follows. We first solve a corresponding free boundary problem in the $(\alpha, \beta)$-plane. This problem is good: the system is linear, the boundary conditions are Lipschitz, but the solution's Jacobian vanishes at the angular point (this corresponds to the phenomenon that the solution of the original problem blows up at the angular point in the $(t, x)$ plane). Then we prove the solution's Jacobian does not vanish in the angular domain, except at the angular point, and then by the inverse mapping theory, we construct the solution of the original problem.

5.1. Some basic facts on classical discontinuous solutions. In order to prove Theorem 5.1, we first review some basic facts on classical discontinuous solutions for system (5.1) (see [12]).

If there exist a $C^{1}$ curve $x=x(t)$ and a piecewise $C^{1}$ vector-valued function $(u, v)=(u(t, x), v(t, x))$ in some open region $\mathcal{R}$ in $\mathbb{R}^{+} \times \mathbb{R}$ such that, on both sides of $x=x(t),(u, v)=(u(t, x), v(t, x))$ satisfies system (5.1) in the classical sense, while, on $x=x(t)$, the Rankine-Hugoniot condition

$$
s[u]=[f(u, v)], \quad s[v]=[g(u, v)]
$$

and the Lax entropy condition

$$
s<\lambda_{1}\left(u_{-}, v_{-}\right), \quad \lambda_{1}\left(u_{+}, v_{+}\right)<s<\lambda_{2}\left(u_{+}, v_{+}\right)
$$

hold, where $s=x^{\prime}(t),[\bullet]$ denotes the jump of $\bullet$ across $x=x(t)$, while $\bullet_{ \pm}$are the values of $\bullet$ on the right side and left side of $x=\varphi(t)$ respectively. Such a vectorvalued function $(u, v)=(u(t, x), v(t, x))$ is called a classical discontinuous solution of system (5.1) in the region $\mathcal{R}$, and $x=x(t)$ is called a slow shock.

Similarly, we can define a classical discontinuous solution of system (5.1) with a fast shock in the region $\mathcal{R}$.

In what follows, we only consider the case of the slow shock. For the case of the fast shock, we may carry out a similar discussion. 
Using the Riemann invariants $w$ and $z$, we may rewrite (5.5) as

$$
\frac{d x(t)}{d t}=H_{1}\left(w_{-}, z_{-}, w_{+}, z_{+}\right), \quad H_{2}\left(w_{-}, z_{-}, w_{+}, z_{+}\right)=0,
$$

where $w_{ \pm}=w\left(u_{ \pm}, v_{ \pm}\right), z_{ \pm}=z\left(u_{ \pm}, v_{ \pm}\right)$, and $H_{i}\left(w_{-}, z_{-}, w_{+}, z_{+}\right)(i=1,2)$ are $C^{4}$ functions of $\left(w_{-}, z_{-}, w_{+}, z_{+}\right)$. Meanwhile, in the Riemann invariants, (5.6) becomes

$$
s<\lambda_{-}\left(w_{-}, z_{-}\right), \quad \lambda_{-}\left(w_{+}, z_{+}\right)<s<\lambda_{+}\left(w_{+}, z_{+}\right) .
$$

The following lemma comes from Theorem 8.6 in [12].

Lemma 5.1. Under the hypotheses (5.3)-(5.4), if $|[z]|$ is small, then

$$
[w]=W\left(w_{ \pm}, z_{ \pm}\right)[z]^{3}
$$

where $W=W\left(w_{ \pm}, z_{ \pm}\right)$is a $C^{4}$ function of $\left(w_{ \pm}, z_{ \pm}\right)$.

Corollary 5.1. Under the hypotheses of Lemma 5.1,

$$
w_{+}=W_{+}\left(w_{-}, z_{ \pm}\right),
$$

where $W_{+}=W_{+}\left(w_{-}, z_{ \pm}\right)$is a $C^{4}$ function of $\left(w_{-}, z_{ \pm}\right)$.

Remark 5.3. In fact, under the assumptions of Lemma 5.1, (5.7) $2,(5.9)$ and (5.10) are all equivalent.

5.2. Proof of Theorem 5.1. It is well known that, if system (5.1) has a $C^{k}$ $(k \geq 1)$ solution on some open domain in $\mathbb{R}^{+} \times \mathbb{R}$, then the corresponding canonical system in characteristic coordinates $(\alpha, \beta)$

$$
\begin{cases}\frac{\partial x}{\partial \alpha}=\lambda_{-}(w, z) \frac{\partial t}{\partial \alpha}, & \frac{\partial z}{\partial \alpha}=0 \\ \frac{\partial x}{\partial \beta}=\lambda_{+}(w, z) \frac{\partial t}{\partial \beta}, & \frac{\partial w}{\partial \beta}=0\end{cases}
$$

also has a $C^{k}(k \geq 1)$ solution $(t, x, w, z)=(t(\alpha, \beta), x(\alpha, \beta), w(\alpha, \beta), z(\alpha, \beta))$ on the corresponding domain in $\mathbb{R} \times \mathbb{R}$. Conversely, if system (5.11) has a $C^{k}(k \geq 1)$ solution $(t, x, w, z)=(t(\alpha, \beta), x(\alpha, \beta), w(\alpha, \beta), z(\alpha, \beta))$ with non-vanishing Jacobian

$$
J=\frac{\partial(t, x)}{\partial(\alpha, \beta)} \neq 0,
$$

then, solving $\alpha, \beta$ from the relation $(t, x)=(t(\alpha, \beta), x(\alpha, \beta))$ and then substituting them into $(w, z)=(w(\alpha, \beta), z(\alpha, \beta))$, we obtain a $C^{k}$ solution of system (5.1) at least in a local domain. System (5.11) has the following advantages:

(i) each equation contains only the derivatives of unknown functions with respect to one independent variable $\alpha$ or $\beta$;

(ii) in the $(\alpha, \beta)$-plane the characteristics are the straight lines: $\alpha=$ const. or $\beta=$ const.

By $\S 4$, system (5.11) has a $C^{4}$ solution in the region $R_{1} R_{7} R_{8} R_{4}$, i.e., $D^{+}$, and in the region $R_{0} R_{4} R_{5} R_{6}$, i.e., $D_{-}$(see Fig. 3). We denote the solutions in $D^{+}$and $D_{-}$by $(t, x, w, z)=\left(t^{+}(\alpha, \beta), x^{+}(\alpha, \beta), w_{0}(\alpha), z_{0}(\beta)\right)$ and $(t, x, w, z)=$ $\left(t_{-}(\alpha, \beta), x_{-}(\alpha, \beta), w_{0}(\alpha), z_{0}(\beta)\right)$ respectively. Moreover, we have

$$
J(\alpha, \beta) \neq 0, \quad \forall(\alpha, \beta) \in \check{\mathrm{D}}^{+} \cup \breve{\mathrm{D}}_{-},
$$

where $\check{\mathrm{D}}^{+} \triangleq D^{+} \backslash\left\{\right.$ point $\left.R_{0}\right\}$ and $\breve{\mathrm{D}}_{-} \triangleq D_{-} \backslash\left\{\right.$ curve $\left.R_{0} R_{6}\right\}$. Furthermore, system (5.1) has a $C^{4}$ solution on $\mho$ and $\Im$ respectively, where $\mho$ denotes the region 
$P_{1} P_{4} P_{8} P_{7} \backslash\left\{\right.$ point $\left.P_{0}\right\}$ and $\Im$ stands for the domain $P_{0} P_{4} P_{5} P_{6} \backslash\left\{\right.$ curve $\left.P_{0} P_{6}\right\}$ (see Fig. 4). We denote the solutions on $\mho$ and $\Im$ by $(w, z)=\left(w_{r}(t, x), z_{r}(t, x)\right)$ and $(w, z)=\left(w_{-}(t, x), z_{-}(t, x)\right)$ respectively.

From now on, we image that there is a strictly decreasing $C^{1}$ curve $x=x(t)(t>$ $t_{0}$ ) issuing from the blow-up point $\left(t_{0}, x_{0}\right)$ (i.e., $\left.x\left(t_{0}\right)=x_{0}\right)$, which is in the open domain bounded by $\gamma^{l}$ and $\gamma^{r}$ (see Fig. 4, in which $P_{0} P_{*}$ stands for $x=x(t)$ ). Let $\nabla_{\left(t_{0}, x_{0}\right)}$ be the set $\left\{(t, x) \mid t_{0}<t \leq t_{0}+\delta_{0}, x(t) \leq x \leq x_{+}\left(t, \alpha_{0}\right)\right\}$, where $\delta_{0}>0$ is a small number. In order to prove the existence in Theorem 5.1, it suffices to solve the following free boundary problem with one characteristic boundary:

$$
\left\{\begin{array}{l}
\frac{\partial w}{\partial t}+\lambda_{+}(w, z) \frac{\partial w}{\partial x}=0, \\
\frac{\partial z}{\partial t}+\lambda_{-}(w, z) \frac{\partial z}{\partial x}=0,
\end{array} \quad \forall(t, x) \in \nabla_{\left(t_{0}, x_{0}\right)} ;\right.
$$

on the free boundary $x=x(t)\left(t>t_{0}\right),(5.7)-(5.8)$ hold, i.e.,

$$
\begin{gathered}
\frac{d x(t)}{d t}=H_{1}\left(w_{-}, z_{-}, w, z\right), \quad H_{2}\left(w_{-}, z_{-}, w, z\right)=0, \\
x^{\prime}(t)<\lambda_{-}\left(w_{-}, z_{-}\right), \quad \lambda_{-}(w, z)<x^{\prime}(t)<\lambda_{+}(w, z) ;
\end{gathered}
$$

on the characteristic boundary $x=x_{+}\left(t, \alpha_{0}\right)\left(t>t_{0}\right)$, we have

$$
w=w_{0}\left(\alpha_{0}\right), \quad z=z_{r}(t, x) .
$$

By Corollary 5.1, we may rewrite the second equality in (5.15) as

$$
w=W_{+}\left(w_{-}(t, x), z_{-}(t, x), z\right),
$$

where $W_{+}=W_{+}\left(w_{-}, z_{-}, z\right)$ is a $C^{4}$ function of $\left(w_{-}, z_{-}, z\right)$.

Unlike the usual free boundary problem, in (5.15)-(5.16) $z_{-}(t, x)$ and $z_{r}(t, x)$ blow up as $(t, x) \rightarrow\left(t_{0}, x_{0}\right)$; that is, it is not Lipschitz continuous at $\left(t_{0}, x_{0}\right)$. To overcome this difficulty, we should ask the aid of system (5.11) and make use of the advantages of system (5.11) sufficiently. Precisely speaking, we first solve a corresponding free boundary problem for system $(5.11)$ in the $(\alpha, \beta)$-plane, then obtain the solution of the problem (5.14)-(5.16) by means of the inverse of the mapping defined by $(t, x)=(t(\alpha, \beta), x(\alpha, \beta))$, where $t=t(\alpha, \beta)$ and $x=x(\alpha, \beta)$ are the first two components of the solution $(t, x, w, z)=(t(\alpha, \beta), x(\alpha, \beta), w(\alpha, \beta), z(\alpha, \beta))$ of the corresponding free boundary problem for system (5.11) in the $(\alpha, \beta)$-plane. In order to get the corresponding problem in the $(\alpha, \beta)$-plane, we first assume that there exists a smooth shock $x=x(t)\left(t \in\left[t_{0}, t_{0}+\delta_{0}\right]\right)$ such that the problem (5.14)(5.16) has a smooth solution $(w, z)=(w(t, x), z(t, x))$ on the domain $\bigcirc_{\left(t_{0}, x_{0}\right)}$; then we derive its corresponding form in the $(\alpha, \beta)$-plane.

Suppose the problem (5.14)-(5.16) has a $C^{4}$ solution $(w, z)=(w(t, x), z(t, x))$ on the domain $\nabla_{\left(t_{0}, x_{0}\right)}$; then system (5.11) also admits a $C^{4}$ solution, denoted by $(t, x, w, z)=\left(t_{+}(\alpha, \beta), x_{+}(\alpha, \beta), w_{+}(\alpha, \beta), z_{+}(\alpha, \beta)\right)$, on the domain $\mathbf{Q}_{\left(\alpha_{0}, \beta_{0}\right)} \triangleq$ $\Pi_{+}^{-1}\left(\bigcirc_{\left(t_{0}, x_{0}\right)}\right)$, where $\Pi_{+} \triangleq\left(t_{+}(\alpha, \beta), x_{+}(\alpha, \beta)\right)$.

Lemma 5.2. Suppose that (5.3)-(5.4) and (4.1)-(4.2) hold. Then the preimage of $x=x(t)\left(t \in\left[t_{0}, t_{0}+\delta_{0}\right]\right)$ under the mapping $\Pi_{-} \triangleq\left(t_{-}(\alpha, \beta), x_{-}(\alpha, \beta)\right)$ can be expressed as

$$
\alpha=\alpha_{-}(\beta), \quad \forall \beta \in\left[\beta_{0}-\varepsilon_{0}, \beta_{0}\right],
$$




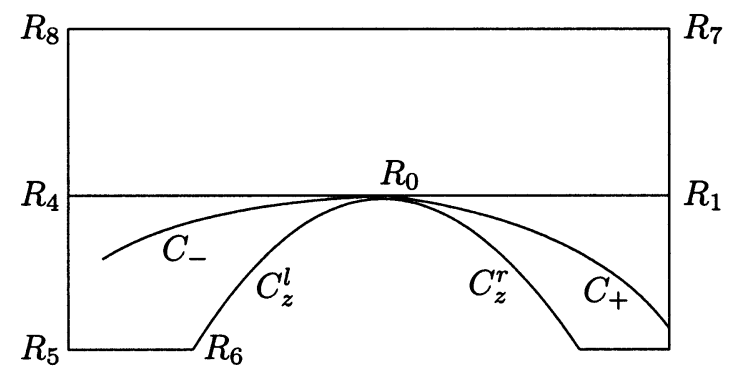

Figure 6. Preimages $C_{ \pm}$of $x=x(t)$ in $(\alpha, \beta)$-plane

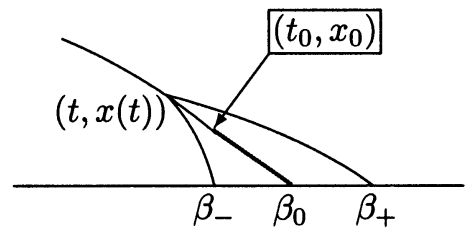

FIgURE 7. Two slow characteristics passing through a point on shock

where $\varepsilon_{0}>0$ is a small number, and $\alpha=\alpha_{-}(\beta)$ is a smooth function satisfying

$$
\alpha_{-}\left(\beta_{0}\right)=\alpha_{0}, \quad \alpha_{-}^{\prime}\left(\beta_{0}\right)=0, \quad \alpha_{-}^{\prime \prime}\left(\beta_{0}\right)=-c_{0}<0,
$$

in which

$$
c_{0}=\frac{\left(\lambda_{+}\left(w_{0}\left(\alpha_{0}\right), z_{0}\left(\beta_{0}\right)\right)-\lambda_{-}\left(w_{0}\left(\alpha_{0}\right), z_{0}\left(\beta_{0}\right)\right)\right)^{-1} t_{\beta \beta \beta}\left(\alpha_{0}, \beta_{0}\right)}{\frac{\partial \lambda_{-}}{\partial z}\left(w_{0}\left(\alpha_{0}\right), z_{0}\left(\beta_{0}\right)\right) z_{0}^{\prime}\left(\beta_{0}\right) t_{\alpha}\left(\alpha_{0}, \beta_{0}\right)}>0 .
$$

Similarly, under the mapping $\Pi_{+}$it can be expressed as

$$
\alpha=\alpha_{+}(\beta), \quad \forall \beta \in\left[\beta_{0}, \beta_{0}+\varepsilon_{0}\right],
$$

where $\alpha=\alpha_{+}(\beta)$ is a smooth function satisfying

$$
\alpha_{+}\left(\beta_{0}\right)=\alpha_{0}, \quad \alpha_{+}^{\prime}\left(\beta_{0}\right)=0, \quad \alpha_{+}^{\prime \prime}\left(\beta_{0}\right)=-c_{0}<0 .
$$

See Fig. 6, in which $C_{ \pm}$stand for the curves $\alpha=\alpha_{ \pm}(\beta)$ respectively.

Proof. Notice that $x=x(t)\left(t \in\left(t_{0}, t_{0}+\delta_{0}\right]\right)$ is a shock growing out of the blow-up point $\left(t_{0}, x_{0}\right)$. By the Lax entropy condition, for any point $(t, x(t))$ on this shock we may draw two slow characteristics, which intersect the $x$-axis at $\left(0, \beta_{-}\right)$and $\left(0, \beta_{+}\right)$ respectively (see Fig. 7). Let $\alpha_{-}$and $\alpha_{+}$denote the corresponding $\alpha$-coordinates of these intersection points respectively.

We only prove (5.18)-(5.19). The proof of (5.21)-(5.22) is similar.

Since $(t, x)=\left(t_{-}\left(\alpha_{-}, \beta_{-}\right), x_{-}\left(\alpha_{-}, \beta_{-}\right)\right)$with $\left(t_{0}, x_{0}\right)=\left(t_{-}\left(\alpha_{0}, \beta_{0}\right), x_{-}\left(\alpha_{0}, \beta_{0}\right)\right)$, on $x=x(t)$ we have

$$
x_{-}\left(\alpha_{-}, \beta_{-}\right)=x\left(t_{-}\left(\alpha_{-}, \beta_{-}\right)\right),
$$

where $\beta_{-} \leq \beta_{0}$ and $\alpha_{-} \leq \alpha_{0}$ (because of (5.8)). Let

$$
F\left(\alpha_{-}, \beta_{-}\right)=x_{-}\left(\alpha_{-}, \beta_{-}\right)-x\left(t_{-}\left(\alpha_{-}, \beta_{-}\right)\right) .
$$


Differentiating (5.24) with respect to $\alpha_{-}$and using (5.11), we get

$$
F_{\alpha_{-}}=\left[\lambda_{-}\left(w_{0}\left(\alpha_{-}\right), z_{0}\left(\beta_{-}\right)\right)-x^{\prime}\left(t_{-}\left(\alpha_{-}, \beta_{-}\right)\right)\right] \frac{\partial t_{-}}{\partial \alpha_{-}} .
$$

Then, by (4.1), Remark 4.1 and the first inequality in (5.8), we obtain

$$
F_{\alpha_{-}}\left(\alpha_{-}, \beta_{-}\right)<0, \quad \forall \beta_{-} \in\left[\beta_{0}-\varepsilon_{0}, \beta_{0}\right),
$$

where $\varepsilon_{0}>0$ is a small number. Hence, solving for $\alpha_{-}$from $F\left(\alpha_{-}, \beta_{-}\right)=0$, we get the desired (5.18).

$(5.19)_{1}$ is obvious. $(5.19)_{2}$ and $(5.19)_{3}$ can be proved in a way similar to the proof of (4.14). The proof is complete.

As before, we know that $\left(w_{-}(t, x), z_{-}(t, x)\right)$ is not Lipschitz continuous at $\left(t_{0}, x_{0}\right)$, but $(t, x)=\left(t_{+}\left(\alpha_{+}, \beta_{+}\right), x_{+}\left(\alpha_{+}, \beta_{+}\right)\right)$is $C^{4}$ smooth on $\overline{\boldsymbol{Q}_{\left(\alpha_{0}, \beta_{0}\right)}}$. Denote

$$
\left\{\begin{array}{l}
\tilde{w}_{-}\left(\alpha_{+}\left(\beta_{+}\right), \beta_{+}\right)=w_{-}\left(t_{+}\left(\alpha_{+}\left(\beta_{+}\right), \beta_{+}\right), x_{+}\left(\alpha_{+}\left(\beta_{+}\right), \beta_{+}\right)\right), \\
\tilde{z}_{-}\left(\alpha_{+}\left(\beta_{+}\right), \beta_{+}\right)=z_{-}\left(t_{+}\left(\alpha_{+}\left(\beta_{+}\right), \beta_{+}\right), x_{+}\left(\alpha_{+}\left(\beta_{+}\right), \beta_{+}\right)\right),
\end{array}\right.
$$

where $\alpha_{+}(\beta)$ is given by (5.21).

Lemma 5.3. Suppose that (5.3)-(5.4) and (4.1)-(4.2) hold. Then

$$
\tilde{w}_{-}\left(\alpha_{+}\left(\beta_{+}\right), \beta_{+}\right) \in C^{4}\left(\left[\beta_{0}, \beta_{0}+\varepsilon_{0}\right]\right), \quad \tilde{z}_{-}\left(\alpha_{+}\left(\beta_{+}\right), \beta_{+}\right) \in C^{4}\left(\left[\beta_{0}, \beta_{0}+\varepsilon_{0}\right]\right) .
$$

Corollary 5.2. It follows from (5.17) that

$$
w=W_{+}\left(\tilde{w}_{-}\left(\alpha_{+}\left(\beta_{+}\right), \beta_{+}\right), \tilde{z}_{-}\left(\alpha_{+}\left(\beta_{+}\right), \beta_{+}\right), z\right),
$$

where $W_{+}$is $C^{4}$ smooth with respect to $\beta_{+} \in\left[\beta_{0}, \beta_{0}+\varepsilon_{0}\right]$.

Proof of Lemma 5.3. We only prove $(5.26)_{2}$. The proof of $(5.26)_{1}$ is similar.

Noting that $z_{-}(t, x(t))=z_{0}\left(\beta_{-}\right)$and using Lemma 5.2 , in order to prove $(5.26)_{2}$ we only need to prove that $\beta_{-}$is a $C^{4}$ function of $\beta_{+}$, where $\beta_{+} \in\left[\beta_{0}, \beta_{0}+\varepsilon_{0}\right]$.

On the shock, we have

$$
T\left(\beta_{-}\right) \triangleq t_{-}\left(\alpha_{-}\left(\beta_{-}\right), \beta_{-}\right)=t_{+}\left(\alpha_{+}\left(\beta_{+}\right), \beta_{+}\right) .
$$

It follows from (4.1), Remark 4.1 and (5.19) that $T^{\prime}\left(\beta_{0}\right)=0$. Moreover, we obtain from (4.1)-(4.2), Remark 4.1 and (5.19) that $T^{\prime \prime}\left(\beta_{0}\right)>0$. This means that $T=$ $T\left(\beta_{-}\right)$is strictly decreasing in $\left[\beta_{0}-\varepsilon_{0}, \beta_{0}\right]$. Therefore, solving for $\beta_{-}$from $(5.27)$ and noting that $t_{ \pm}=t_{ \pm}\left(\alpha_{ \pm}, \beta_{ \pm}\right)$are $C^{4}$ functions of $\beta_{ \pm}$, we see that $\beta_{-}$is a $C^{4}$ function of $\beta_{+}$. This completes the proof.

Set

$$
\nabla_{\left(\alpha_{0}, \beta_{0}\right)}=\left\{(\alpha, \beta) \mid \beta_{0} \leq \beta \leq \beta_{0}+\varepsilon_{0}, \varrho(\beta) \leq \alpha \leq \alpha_{0}\right\},
$$

where $\varepsilon_{0}>0$ is a small number and $\varrho(\beta)$ is a free boundary with

$$
\varrho\left(\beta_{0}\right)=\alpha_{0}, \quad \varrho^{\prime}\left(\beta_{0}\right)=0, \quad \varrho^{\prime \prime}\left(\beta_{0}\right)=-c_{0}<0,
$$

where $c_{0}$ is defined by (5.20). See Fig. 8 . In $\diamond\left(\alpha_{0}, \beta_{0}\right)$, we consider the free boundary problem for system (5.11) with the following boundary conditions: on $\alpha=\varrho(\beta)$,

$$
\begin{aligned}
& d x=H_{1}\left(\bar{w}_{-}(\varrho(\beta), \beta), \bar{z}_{-}(\varrho(\beta), \beta), w, z\right) d t, \\
& w=W_{+}\left(\bar{w}_{-}(\varrho(\beta), \beta), \bar{z}_{-}(\varrho(\beta), \beta), z\right),
\end{aligned}
$$




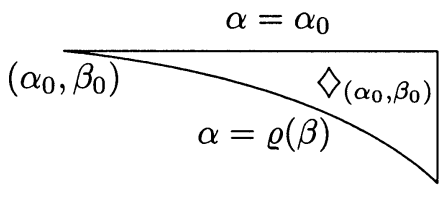

FiguRE 8. Domain $\diamond_{\left(\alpha_{0}, \beta_{0}\right)}$ in $(\alpha, \beta)$-plane

where $\bar{w}_{-}, \bar{z}_{-}$are $C^{4}$ functions defined by the solution in $D_{-}$; on $\alpha=\alpha_{0}$,

$$
t=t^{+}\left(\alpha_{0}, \beta\right), x=x^{+}\left(\alpha_{0}, \beta\right), w=w_{0}\left(\alpha_{0}\right), z=z_{0}(\beta) ;
$$

moreover, at $\left(\alpha_{0}, \beta_{0}\right)$

$$
\begin{aligned}
& t_{\alpha}=t_{\alpha}\left(\alpha_{0}, \beta_{0}\right), \quad x_{\alpha}=x_{\alpha}\left(\alpha_{0}, \beta_{0}\right), \quad z=z_{0}\left(\beta_{0}\right), \\
& t_{\beta}=t_{\beta}\left(\alpha_{0}, \beta_{0}\right), \quad x_{\beta}=x_{\beta}\left(\alpha_{0}, \beta_{0}\right), \quad w=w_{0}\left(\alpha_{0}\right) .
\end{aligned}
$$

Remark 5.4. Here we would like to emphasize that $H_{1}\left(\bar{w}_{-}, \bar{z}_{-}, w, z\right)$ is a $C^{4}$ function of $\left(\bar{w}_{-}, \bar{z}_{-}, w, z\right), W_{+}\left(\bar{w}_{-}, \bar{z}_{-}, z\right)$ is a $C^{4}$ function of $\left(\bar{w}_{-}, \bar{z}_{-}, z\right), \bar{w}_{-}(\varrho(\beta), \beta)$ and $\bar{z}_{-}(\varrho(\beta), \beta)$ are $C^{4}$ functions of $\beta$, while $t^{+}$and $x^{+}$are $C^{4}$ functions of $\beta$. Therefore, they are Lipschitz continuous with respect to all the variables.

By the above discussion, in order to solve the problem (5.14)-(5.16), it suffices to prove that there exists a unique $\alpha=\varrho(\beta)$ satisfying (5.29) such that the problem (5.11), (5.30)-(5.32) possesses a $C^{4}$ solution $(t, x, w, z)=(t(\alpha, \beta), x(\alpha, \beta)$, $w(\alpha, \beta), z(\alpha, \beta))$ with non-vanishing Jacobian

$$
J(\alpha, \beta)=\frac{\partial(t, x)}{\partial(\alpha, \beta)} \neq 0, \quad \forall(\alpha, \beta) \in \diamond_{\left(\alpha_{0}, \beta_{0}\right) \backslash\left\{\left(\alpha_{0}, \beta_{0}\right)\right\} .}
$$

Lemma 5.4. Under the hypotheses (5.3)-(5.4) and (4.1)-(4.2), if the problem (5.11), (5.30)-(5.32) has a $C^{1}$ solution $(t, x, w, z)=(t(\alpha, \beta), x(\alpha, \beta), w(\alpha, \beta)$, $z(\alpha, \beta))$ in $\nabla_{\left(\alpha_{0}, \beta_{0}\right)}$, then (5.33) always holds.

Proof. It follows from (4.17) that

$$
t_{\alpha}\left(\alpha_{0}, \beta\right)<0, \quad t_{\beta}\left(\alpha_{0}, \beta\right)>0, \quad \forall \beta \in\left(\beta_{0}, \beta_{0}+\varepsilon_{0}\right] .
$$

Then by (2.9), in order to prove (5.33), it suffices to show that

$$
t_{\alpha}(\alpha, \beta)<0, \quad t_{\beta}(\alpha, \beta)>0, \quad \forall(\alpha, \beta) \in \diamond_{\left(\alpha_{0}, \beta_{0}\right) \backslash\left\{\left(\alpha_{0}, \beta_{0}\right)\right\} .}
$$

The first inequality comes directly from the first inequality in (4.1) (because of Remark 4.1), provided that $\varepsilon_{0}>0$ is small enough.

It remains to prove the second inequality in (5.35).

We first claim that

$$
\left.t_{\beta}(\alpha, \beta)\right|_{\alpha=\varrho(\beta)}>0, \quad \forall \beta \in\left(\beta_{0}, \beta_{0}+\varepsilon_{0}\right] .
$$

In fact, it follows from $(5.30)_{1}$ that, on $\alpha=\varrho(\beta)$,

$$
x_{\alpha} \varrho^{\prime}(\beta)+x_{\beta}=H_{1}\left(t_{\alpha} \varrho^{\prime}(\beta)+t_{\beta}\right) .
$$

Using (5.11), we get

$$
\left(\lambda_{-}^{r}-H_{1}\right) \varrho^{\prime}(\beta) t_{\alpha}=\left(H_{1}-\lambda_{+}^{r}\right) t_{\beta},
$$

where $\lambda_{ \pm}^{r}=\lambda_{ \pm}(w(\varrho(\beta), \beta), z(\varrho(\beta), \beta))$. Then, noting (5.8) and (5.29) and using the first inequality in (5.35), we obtain (5.36). 
Similarly to (4.20), for any $(\alpha, \beta) \in \diamond_{\left(\alpha_{0}, \beta_{0}\right)} \backslash\left\{\left(\alpha_{0}, \beta_{0}\right)\right\}$ we have

$$
t_{\beta}(\alpha, \beta)=\left.t_{\beta}(\alpha, \beta)\right|_{\alpha=\varrho(\beta)}+\int_{\varrho(\beta)}^{\alpha} G(\zeta, \beta) \exp Q(\zeta, \alpha, \beta) d \zeta .
$$

Noting (5.36), we get the second inequality in (5.35). The proof is complete.

Lemma 5.5. Under the hypotheses (5.3)-(5.4) and (4.1)-(4.2), there is a $C^{4}$ smooth curve $\alpha=\varrho(\beta)$ satisfying (5.29) such that the problem (5.11), (5.30)-(5.32) has a unique $C^{4}$ solution $(t, x, w, z)=(t(\alpha, \beta), x(\alpha, \beta), w(\alpha, \beta), z(\alpha, \beta))$.

Proof. In order to avoid the trouble introduced by the free boundary $\alpha=\varrho(\beta)$, we make the following transformation:

$$
\tilde{\alpha}=-\frac{c_{0}\left(\alpha-\alpha_{0}\right)(1+\varrho(\beta)-\alpha)}{2\left(\varrho(\beta)-\alpha_{0}\right)}\left(\beta-\beta_{0}\right)^{2}, \quad \tilde{\beta}=\beta-\beta_{0} .
$$

Under (5.39), the free boundary $\alpha=\varrho(\beta)$ reduces to a fixed boundary $\tilde{\alpha}=-\frac{c_{0}}{2} \tilde{\beta}^{2}$ in the new variables $(\tilde{\alpha}, \tilde{\beta})$; moreover, the fixed boundary keeps the geometrical properties of $\alpha=\varrho(\beta)$ at $\left(\alpha_{0}, \beta_{0}\right)$. However, in $(\tilde{\alpha}, \tilde{\beta})$-space the original free boundary problem (5.11), (5.30)-(5.32) becomes a fixed boundary value problem for a quasilinear hyperbolic system with boundary conditions in functional form. It is easy to see that this kind of fixed boundary value problem is equivalent to a system of integral equations with some undetermined coefficients (see [15]). In a manner similar to [15], we can prove that the system of integral equations has a unique $C^{4}$ solution at least in a local domain $\left\{(\tilde{\alpha}, \tilde{\beta}) \mid-\frac{c_{0}}{2} \tilde{\beta}^{2} \leq \tilde{\alpha} \leq 0,0 \leq \tilde{\beta} \leq \varepsilon_{0}\right\}$, provided that $\varepsilon_{0}>0$ is sufficiently small. Noting (5.39), we see that there exists a function $\alpha=\varrho(\beta)$ satisfying (5.29) such that the problem (5.11), (5.30)-(5.32) has a unique $C^{4}$ solution in $\nabla_{\left(\alpha_{0}, \beta_{0}\right)}$. Because of space limitations, we omit the details.

Proof of Theorem 5.1. The existence of a classical discontinuous solution follows from Lemmas 5.4-5.5 easily. In particular, by the process of our construction for the classical discontinuous solution, the Lax entropy condition on shock is satisfied automatically, and the solution is continuous across the characteristic $x=x_{+}\left(t, \alpha_{0}\right)\left(t \geq t_{0}\right)$. However, we do not know if its derivatives are continuous across this characteristic. Therefore this characteristic might be a weak discontinuity issuing from the blow-up point $\left(t_{0}, x_{0}\right)$.

The uniqueness comes from Liu [16] directly. The proof is complete.

\section{ACKNOWLEDGEMENT}

The author thanks Professor B. L. Keyfitz for kind support and encouragement, and the referee for pertinent comments and valuable suggestions.

\section{REFERENCES}

[1] S. Alinhac, Blowup for Nonlinear Hyperbolic Equations, Progress in Nonlinear Differential Equations and Their Applications 17, Birkhäuser, 1995. MR 96h:35109

[2] R. Bryant, P. Griffiths and L. Hsu, Toward a geometry of differential equations, Geometry, Topology, and Physics for Raoul Bott, ed. by S. T. Yau, International Press, 1994, pp. 1-76. MR 97b:58005

[3] P. H. Chang, On the breakdown phenomena of solutions of quasilinear wave equations, Michigan Math. J. 23 (1976), 277-287. MR 57:901

[4] Shu-xing Chen and Li-ming Dong, Formation of shocks for p-system with general smooth initial data, to appear. 
[5] J. Guckenheimer, Solving a single conservation law, Lect. Notes Math. 468, Springer-Verlag, 1975, pp. 108-134. MR 58:29336

[6] L. Hörmander, Lectures on Nonlinear Hyperbolic Differential Equations, Mathématiques et Applications 26, Springer, 1997. MR 98e:35103

[7] G. Jennings, Piecewise smooth solutions of a single conservation law exist, Adv. in Math. 33 (1979), 192-205. MR 80j:35067

[8] F. John, Formation of singularities in one-dimensional nonlinear wave propagation, Comm. Pure Appl. Math. 27 (1974), 377-405. MR 51:6163

[9] J. B. Keller and L. Ting, Periodic vibration of systems governed by nonlinear partial differential equations, Comm. Pure Appl. Math. 19 (1966), 371-420. MR 34:5347

[10] De-xing Kong, Cauchy Problem for Quasilinear Hyperbolic Systems, MSJ Memoirs 6, The Mathematical Society of Japan, Tokyo, 2000. MR 2002b:35127

[11] De-xing Kong, Life-span of classical solutions to quasilinear hyperbolic systems with slow decay initial data, Chinese Ann. of Math. 21B (2000), 413-440. MR 2001j:35186

[12] P. D. Lax, Hyperbolic systems of conservation laws II, Comm. Pure Appl. Math. 10 (1957), 537-566. MR 20:176

[13] M. P. Lebaud, Description de le formation d'un choc dans le p-système, J. Math. Pures Appl. 73 (1994), 523-565. MR 96a:35115

[14] Li Ta-tsien, Global Classical Solutions for Quasilinear Hyperbolic Systems, Research in Applied Mathematics 32, Wiley-Masson, 1994. MR 95m:35115

[15] Li Ta-tsien and Yu Wen-ci, Boundary Value Problems for Quasilinear Hyperbolic Systems, Duke University Mathematics Series V, Duke University, 1985. MR 88g:35115

[16] Liu Tai-ping, Uniqueness of weak solutions of the Cauchy problem for general $2 \times 2$ conservation laws, J. Differential Equations 20 (1976), 369-388. MR 52:14678

[17] R. C. MacCamy and V. J. Mizel, Existence and nonexistence in the large of solutions of quasilinear wave equations, Arch. Rat. Mech. Anal. 25 (1967), 299-320. MR 35:7000

[18] S. Nakane, Formation of shocks for a single conservation law, SIAM J. Math. Anal. 19 (1988), 1391-1408. MR 89k:35142

[19] D. G. Schaeffer, A regularity theorem for conservation laws, Adv. in Math. 11 (1973), 368386. MR 48:4523

[20] H. Whitney, On singularities of mappings of Euclidean space. I: Mappings of the plane into the plane, Ann. of Math. 62 (1955), 374-410. MR 17:518d

Department of Applied Mathematics, Shanghai Jiao Tong University, Shanghai 200030, CHINA

E-mail address: dkong@mail.sjtu.edu.cn 